\title{
1 Helium in the eroding atmosphere of an exoplanet
}

2 J. J. Spake ${ }^{1}$, D. K. Sing ${ }^{1,2}$, T. M. Evans ${ }^{1}$, A. Oklopčić ${ }^{3}$, V. Bourrier ${ }^{4}$, L. Kreidberg ${ }^{5,6}$, B. V.

3 Rackham $^{7,8,9}$, J. Irwin ${ }^{6}$, D. Ehrenreich ${ }^{4}$, A. Wyttenbach ${ }^{4}$, H. R. Wakeford ${ }^{11}$, Y. Zhou ${ }^{7}$, K. L.

4 Chubb $^{10}$, N. Nikolov ${ }^{1}$, J. Goyal $^{1}$, G. W. Henry ${ }^{12}$, M. H. Williamson ${ }^{12}$, S. Blumenthal ${ }^{1}$, D.

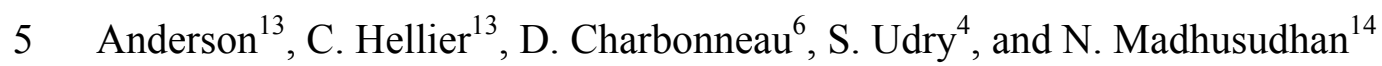

$6{ }^{I}$ Astrophysics Group, School of Physics, University of Exeter, Stocker Road, Exeter, EX4 4QL, UK.

$7 \quad{ }^{2}$ Department of Earth and Planetary Sciences, Johns Hopkins University, Baltimore, MD, USA

$8{ }^{3}$ Institute for Theory and Computation, Harvard-Smithsonian Center for Astrophysics 60 Garden Street, MS-51, Cambridge,

9 Massachusetts 02138 , USA

$10{ }^{4}$ Observatoire de l'Université de Genève, 51 chemin des Maillettes, 1290 Sauverny, Switzerland

$11{ }^{5}$ Harvard Society of Fellows 78 Mt. Auburn St. Cambridge, MA 02138, USA

$12{ }^{6}$ Harvard-Smithsonian Center for Astrophysics 60 Garden St. Cambridge, MA 02138

$13{ }^{7}$ Department of Astronomy/Steward Observatory, The University of Arizona, 933 N. Cherry Avenue, Tucson, AZ 85721, USA

$14{ }^{8}$ National Science Foundation Graduate Research Fellow

$15{ }^{9}$ Earths in Other Solar Systems Team, NASA Nexus for Exoplanet System Science

${ }^{10}$ Department of Physics and Astronomy, University College London, London, WC1E 6BT, UK

$17{ }^{11}$ Space Telescope Science Institute, 3700 San Martin Drive, Baltimore, MD 21218, USA

$18{ }^{12}$ Center of Excellence in Information Systems, Tennessee State University,, Nashville, TN 37209, USA

$19{ }^{13}$ Astrophysics Group, Keele University, Staffordshire, ST5 5BG, UK

$20{ }^{14}$ Institute of Astronomy, University of Cambridge, Madingley Road, Cambridge CB3 OHA, UK 
Helium is the second most abundant element in the universe after hydrogen and is a major constituent of gas-giant planets in our Solar System. Early theoretical models predicted helium to be among the most readily-detectable species in the atmospheres of exoplanets, especially in extended and escaping atmospheres ${ }^{1}$. However, searches for helium have until now been unsuccessful ${ }^{2}$. Here we present the first detection of helium on an exoplanet, at a confidence level of $4.5 \sigma$. We measured the near-infrared transmission spectrum of the warm gas giant WASP-107b ${ }^{3}$ with the Hubble Space

Telescope and identified the narrow absorption feature of excited, metastable helium at 10,833 angstroms. The amplitude of the feature, in transit depth, is $0.049 \pm 0.011 \%$ in a bandpass of 98 angstroms, which is more than 5 times greater than that which could be caused by nominal stellar chromospheric activity. The large absorption signal suggests that WASP-107b has an extended atmosphere that is eroding at a total rate of $10^{10}$. $3 \times 10^{11} \mathrm{~g} \mathrm{~s}^{-1}(0.1-4 \%$ of its total mass per Gyr), and may have a comet-like tail of gas shaped by radiation pressure. WASP-107b is one of the lowest density planets known, with a radius similar to that of Jupiter $\left(0.94 \pm 0.02 \mathrm{R}_{\mathrm{J}}\right)$ and a much lower mass $\left(0.12 \pm 0.01 \mathrm{M}_{\mathrm{J}}\right)^{3}$. It orbits an active K6 dwarf the Hubble Space Telescope (HST). Our observations lasted 7 hours and we acquired 84 time-series spectra with the G102 grism, which covers the 8,000-11,000 $\AA$ wavelength range. Further details of the observations and data reduction can be found in Methods. Each spectrum was integrated along the wavelength axis to first produce a 'white' light curve (Extended Data Fig. 1). In addition to the planetary transit signal, the resulting time series was affected by instrumental systematics caused by electron trapping in the WFC3 detector. 
trend and a physically-motivated WFC3 systematics model ${ }^{15}$. For the planetary transit model, we allowed the planet-to-star radius ratio $\left(R_{p} / R_{s}\right)$ and the mid-transit time $\left(T_{0}\right)$ to vary as free parameters, while holding the ratio of orbital distance to stellar radius $\left(a / R_{s}\right)$, inclination $(i)$, eccentricity $(e)$, and period $(P)$, fixed to previously determined values ${ }^{6,16}$. We assumed a quadratic limb-darkening profile for the star, holding the coefficients fixed to values determined from a model stellar spectrum ${ }^{17}$. Further details of this fit are provided in Methods. The results of the fit are reported in Extended Data Table 1, and Extended Data Fig. 1.

Two sets of spectroscopic light curves were constructed by summing each spectrum into broad- and narrow-band bins. The first set consisted of 9 broad-band channels spanning the 8,770-11,360 A wavelength range, while the second set comprised 20 overlapping, narrowband channels spanning the 10,580-11,070 $\AA$ wavelength range. The narrow-band channels cover the helium absorption triplet at 10,833 $\AA$ (vacuum wavelength - the air wavelength of this line is $10,830 \AA)$. The widths of the broadband and narrowband channels were $294 \AA$ (12 pixel columns) and $98 \AA$ (4 pixel columns), respectively. We fitted both sets of spectroscopic light curves using the same approach as described above for the white light curve. However, for the planetary transit signals, we only allowed $R_{p} / R_{s}$ to vary as a free parameter, while holding $t_{0}, a / R_{s}, i, e$, and $P$ fixed to those reported in Extended Data Table 1. We fixed limb darkening coefficients in a similar way to the white light curve fit. Additional details of the fitting procedure are given in Methods. The inferred values for the transit depth, $\left(R_{p} / R_{s}\right)^{2}$, in each wavelength channel are shown in Fig. 1 and Extended Data Table 2. These results constitute the atmospheric transmission spectrum. The broadband transmission spectrum is consistent with a previous transmission spectrum for WASP-107b obtained using the WFC3 G141 grism, which covers the 11,000-16,000 $\AA$ wavelength range $\mathrm{e}^{18}$. The latter exhibits a muted water absorption band centred at $14,000 \AA$, 
with an otherwise flat spectrum implying an opaque cloud deck. After applying a correction for stellar activity variations between the G102 and G141 observation epochs (see Methods), the G102 spectrum aligns with the cloud deck level inferred from the G141 spectrum (Fig. 1). The helium triplet has an expected width of approximately $3 \AA$, whereas the resolution of the G102 grism is $67 \AA$ ( 3 pixels $)$ at 10,400 $\AA^{19}$. Therefore, to make a finely-sampled transmission spectrum, we shifted each of the 20 narrowband channels by 1 pixel with respect to the adjacent channel along the wavelength axis. The narrowband transmission spectrum peaked when the binning was most closely centred at 10,833 $\AA$ (Figure 3), as expected if absorption by helium in the planetary atmosphere was responsible for the signal. To estimate the amplitude of the absorption feature, we focussed on 5 non-overlapping channels centred on 10,833 $\AA$. All but one of the channels were consistent with a baseline transit depth level of $2.056 \pm 0.005 \%$. The single exception is the channel centred on the $10,833 \AA$ helium triplet, for which the transit is visibly deeper than for the surrounding channels (Fig. 2), and we obtained $\left(R_{p} / R_{s}\right)^{2}=2.105 \pm 0.010 \%$. We ruled out various alternative explanations for the signal, including other absorbing species, helium in the Earth's atmosphere, and the occultation of inhomogeneities in the stellar chromosphere and photosphere (see Methods).

96 The metastable helium probed by $10,833 \AA$ absorption forms high up, at $\mu$ bar - mbar 97 pressures in planetary atmospheres, where stellar XUV radiation is absorbed ${ }^{12}$. On the other hand, absorption of the neighbouring continuum occurs deeper in planetary atmospheres, at mbar - bar pressures. Therefore, to interpret the broadband (continuum) and narrowband $(\sim 10,833 \AA)$ transmission spectra, we used separate lower- and upper- atmosphere models.

101 For the combined G102 and G141 broadband spectrum (with the 10,775-10,873 $\AA$ range removed), we performed an atmospheric retrieval analysis using our one-dimensional radiative transfer code, $\mathrm{ATMO}^{20,21}$ (see Methods and Extended Data Table 3). We found the 
104 broadband data were well explained by a grey absorbing cloud deck across the full 8,780-

$10511,370 \AA$ wavelength range, in addition to $\mathrm{H}_{2} \mathrm{O}$ absorption. We obtained a volume mixing

106 ratio for $\mathrm{H}_{2} \mathrm{O}$ of $5 \times 10^{-3}-4 \times 10^{-2}$, consistent with previous estimations ${ }^{18}$.

107 We investigated the narrowband transmission spectrum using two numerical models for the upper atmosphere of WASP-107b (see Methods). Our first, 1-D model ${ }^{22}$ solves for the level populations of a $\mathrm{H} / \mathrm{He}$ Parker wind, and suggests that WASP-107b is losing its atmosphere at

110 a rate of $10^{10}-3 \times 10^{11} \mathrm{~g} \mathrm{~s}^{-1}$, corresponding to $\sim 0.1-4 \%$ of its total mass every billion years.

111 Our second, 3-D model ${ }^{8,23}$ suggests an escape rate for metastable helium of $10^{6}-10^{7} \mathrm{~g} / \mathrm{s}$ (for

112 comparison, the 1-D model gives an escape rate of $\sim 10^{5} \mathrm{~g} \mathrm{~s}^{-1}$ for $2^{3} \mathrm{~S}$ helium). It also suggests

113 that stellar radiation pressure blows away the escaping helium atoms so swiftly as to form a

114 tail nearly aligned with the star-planet axis, which explains the lack of post-transit occultation

115 detected in our data (Figure 2). The radiation pressure should also blue-shift the absorption

116 signature over hundreds of $\mathrm{km} \mathrm{s}^{-1}$, which may be observable at higher spectral resolution

117 (Fig. 4).

118 Atmospheric mass-loss can substantially alter the bulk composition of a planet. For example,

119 there is evidence that atmospheric escape is responsible for the observed dearth of highly-

120 irradiated super-Earth and sub-Neptune exoplanets with sizes between 1.6 and 2 Earth radii ${ }^{24-}$

$121{ }^{28}$. In order to calibrate theories of planet formation, and assess whether these planets have

122 substantial $\mathrm{H} / \mathrm{He}$ envelopes, it is necessary to understand how atmospheric mass-loss affects

123 the subsequent evolution of bodies that start with significant atmospheres. Empirical

124 constraints such as the one presented here for WASP-107b are therefore crucial for retracing

125 evolutionary pathways and interpreting the present day population of planets ${ }^{29}$.

126 To date, extended atmospheres have been detected on three exoplanets by targeting the

127 Lyman-alpha line in the $\mathrm{UV}^{4,7,8}$, and on one exoplanet using the optical H-alpha line ${ }^{11}$. Our

128 observations of WASP-107b in the $10,833 \AA$ line provide not only the first detection of 
129 helium on an exoplanet, but also the first detection of an extended exoplanet atmosphere at

130 infrared wavelengths. This result demonstrates a new method to study extended atmospheres

131 which is complementary to the two hydrogen lines.

132 We note that observations targeting the 10,833 $\AA$ helium triplet are possible from the ground

133 with existing high-resolution infrared spectrographs. In the near future, high signal-to-noise

134 observations will also be possible with the James Webb Space Telescope at a spectral

135 resolution of $\Delta \lambda \sim 4 \AA\left(\sim 110 \mathrm{kms}^{-1}\right)$.

136

Online Content Methods, along with any additional Extended Data display items and Source Data are available in the online version of the paper; references unique to those section appear only in the online paper.

Received ;

References:

143 1. Seager, S., Sasselov, D. D., Theoretical Transmission Spectra during Extrasolar Giant

144 Planet Transits, Astrophys. J. 537, 916-921 (2000)

145 2. Moutou, C., Coustenis, A., Schneider, J., Queloz, D.; Mayor, M., Searching for helium in

146 the exosphere of HD 209458b, Astron. Astrophys, 405, 341-348 (2003)

147 3. Anderson, D. et al., The discoveries of WASP-91b, WASP-105b and WASP-107b: Two

148 warm Jupiters and a planet in the transition region between ice giants and gas giants, Astron.

149 Astrophys., 604, A110 (2017)

150 4. Vidal-Madjar, A. et al. An extended upper atmosphere around the extrasolar planet

151 HD209458b. Nature 422, 143-146 (2003)

152 5. Vidal-Madjar, A. et al. Detection of oxygen and carbon in the hydrodynamically escaping 153 atmosphere of the extrasolar planet HD 209458b. Astrophys. J, 604, L69-L72 (2004) 
154 6. Fossati, L. et al. Metals in the exosphere of the highly irradiated planet WASP-12b.

155 Astrophys. J, 760, L222 (2010)

156 7. Lecavelier des Etangs, A. et al., Evaporation of the planet HD 189733b observed in H I

157 Lyman- $\alpha$. Astron. Astrophys. 514, A72 (2010).

158 8. Kulow, J. R., France, K., Linsky, J., Loyd, R. O. P., Lya Transit Spectroscopy and the

159 Neutral Hydrogen Tail of the Hot Neptune GJ 436b, Astrophys. J, 786, A132 (2014)

160 9. Ehrenreich, D. et al., A giant comet-like cloud of hydrogen escaping the warm Neptune-

161 mass exoplanet GJ 436b, Nature, 522, 459-461 (2015)

162 10. Winn, J. N., et al., A Search for Ha Absorption in the Exosphere of the Transiting

163 Extrasolar Planet HD 209458b, Publ. Astron. Soc. Jpn, 56, 655-662 (2004)

164 11. Jensen, A. G., et al., A Detection of Ha in an Exoplanetary Exosphere, Astrophys. J., 751, 165 A86, (2012)

166 12. Christie, D., Arras, P., Li, Z., Ha Absorption in Transiting Exoplanet Atmospheres,

167 Astrophys. J., 772, A144, (2013)

168 13. Cauley, P. W., Redfield, S., Jensen, A. G., A Decade of Ha Transits for HD 189733 b:

169 Stellar Activity versus Absorption in the Extended Atmosphere, Astron. J., 153, A217, $170 \quad(2017)$

171 14. Kreidberg, L., batman: Basic Transit Model cAlculatioN in Python, PASP, 127, 1161 172 (2015)

173 15. Zhou, Y., Apai, D., Lew, B. W. P., Schneider, G., A Physical Model-based Correction for 174 Charge Traps in the Hubble Space Telescope's Wide Field Camera 3 Near-IR Detector and 175 Its Applications to Transiting Exoplanets and Brown Dwarfs, Astron. J., 153, 243 (2017)

176 16. Dai, F., Winn, J., The Oblique Orbit of WASP-107b from K2 Photometry, Asron. J., 153, $177 \quad 205(2017)$ 
178 17. Castelli, F., Kurucz, R. L., New Grids of ATLAS9 Model Atmospheres, eprint

179 arXiv:astro-ph/0405087

180 18. Kreidberg, L., Line, M., Thorngren, D., Morley, C., Stevenson, S., Water, Methane

181 Depletion, and High-Altitude Condensates in the Atmosphere of the Warm Super-Neptune

182 WASP-107b, eprint arXiv:1709.08635

183 19. Kuntschner, H., Bushouse, H., Kummel, M., Walsh, J. R., WFC3 SMOV proposal 11552:

184 Calibration of the G102 grism, ST-ECF Instrument Science Report WFC3-2009-18 (2009)

185 20. Amundsen, D., et al., Accuracy tests of radiation schemes used in hot Jupiter global

186 circulation models, Astron. Astrophys, 564, A59 (2014)

187 21. Tremblin, P., et al., Fingering Convection and Cloudless Models for Cool Brown Dwarf

188 Atmospheres, Astrophys. J, 804, L17 (2015)

189 22. Oklopčić, A., Hirata, C., M., A New Window into Escaping Exoplanet Atmospheres:

19010830 AA Line of Metastable Helium, eprint arXiv:1711.05269

191 23. Bourrier, V., Lecavelier des Etangs, A., Ehrenreich., D., Tanaka., Y. A., Vidotto, A. A.,

192 An evaporating planet in the wind: stellar wind interactions with the radiatively braked

193 exosphere of GJ 436 b, Astron. Astrophys, 591, A121 (2016)

194 24. Lopez, E. D., Fortney, J. J., Miller., N., How Thermal Evolution and Mass-loss Sculpt

195 Populations of Super-Earths and Sub-Neptunes: Application to the Kepler-11 System and

196 Beyond, Astrophys. J, 761, A59 (2012)

197 25. Owen, J., Wu, Y., Kepler Planets: A Tale of Evaporation, Astrophys. J, 775, A105 (2013)

198 26. Jin, S. et al., Planetary Population Synthesis Coupled with Atmospheric Escape: A

199 Statistical View of Evaporation, Astrophys. J, 795, A65 (2014)

200 27. Chen, H., Rogers, L. A., Evolutionary Analysis of Gaseous Sub-Neptune-mass Planets

201 with MESA, Astrophys. J, 831, A180 (2016) 

discussions. This work is based on observations with the NASA/ESA HST, obtained at the Space Telescope

210 Science Institute (STScI) operated by AURA, Inc. J.J.S. is supported by an STFC studentship. The research

211 leading to these results has received funding from the European Research Council under the European Union's

212 Seventh Framework Programme (FP7/2007-2013) / ERC grant agreement no. 336792. Support for this work

213 was provided by NASA through grants under the HST-GO-14916 programme from the STScI. G.W.H. and

214 M.H.W. acknowledge support from Tennessee State University and the State of Tennessee through its Centers

215 of Excellence program. The MEarth Team gratefully acknowledges funding from the David and Lucille Packard

216 Fellowship for Science and Engineering, the National Science Foundation, and the John Templeton Foundation.

217 The opinions expressed in this publication are those of the authors and do not necessarily reflect the views of the

218 John Templeton Foundation. This work has been carried out in the frame of the National Centre for Competence

219 in Research PlanetS supported by the Swiss National Science Foundation (SNSF). VB, DE, AW and SU

220 acknowledge the financial support of the SNSF. DE and VB acknowledge funding from the European Research

221 Council (ERC) under the European Union's Horizon 2020 research and innovation programme (project FOUR

222 ACES; grant agreement No 724427)

223 Author Contributions J.J.S. led the HST telescope time proposal, designed the observations, and led the data 224 analysis with contributions from T.M.E., H.R.W., L.K and Y. Z.. J.J.S. identified the planetary helium, and wrote the manuscript with contributions from T.M.E., V.B., A.O., J.I., B.V.R and G.W.H. A.O. and V.B.

226 performed detailed modelling of the exosphere, with contributions from D.E. D.K.S. provided scientific guidance and performed the retrieval analysis. J.I., G.H., M. H. and D.C. provided ground-based 
230 are welcome to comment on the online version of the paper. Correspondence and requests for materials should

231 be addressed to J. J. S. (jspake@astro.ex.ac.uk)

232 Competing interests

233 The authors declare no competing interests.

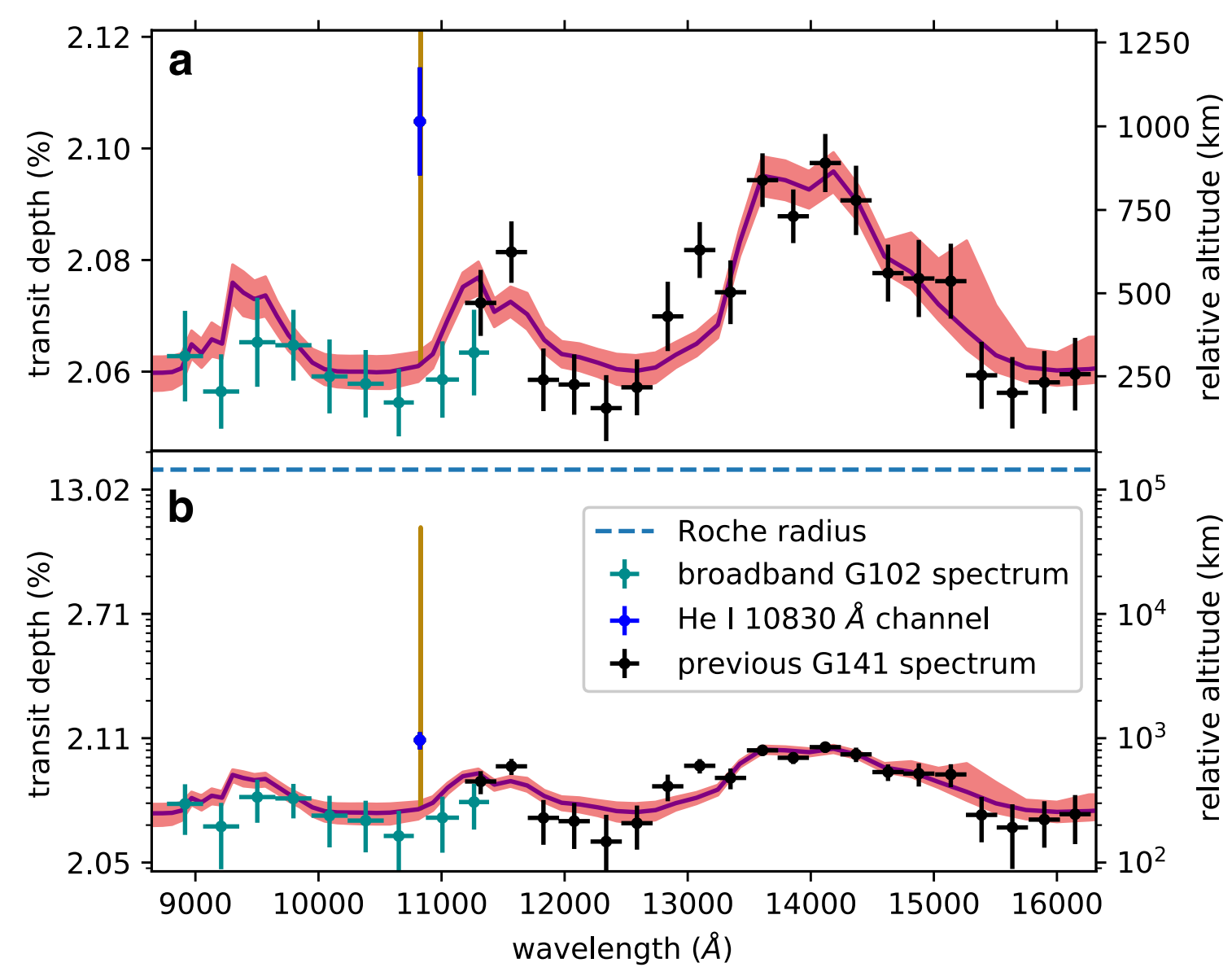

Figure 1 | Combined near-infrared transmission spectrum for WASP-107b with helium

absorption feature. (a) Data plotted on a linear scale. Points with $1 \sigma$ error bars are from a previous study ${ }^{18}$ and this work, both corrected for stellar activity (see Methods). The solid purple line is the best fit lower atmosphere retrieval model from MCMC fits, and the shaded

239 pink areas encompass $68 \%, 95 \%$ and $99.7 \%$ of the MCMC samples. The gold line is the best-

240 fit helium 10,830 $\AA$ absorption profile from our 1-D escaping atmosphere model. (b) Same as

241 (a), on a log scale. The dashed blue line shows the Roche radius. 


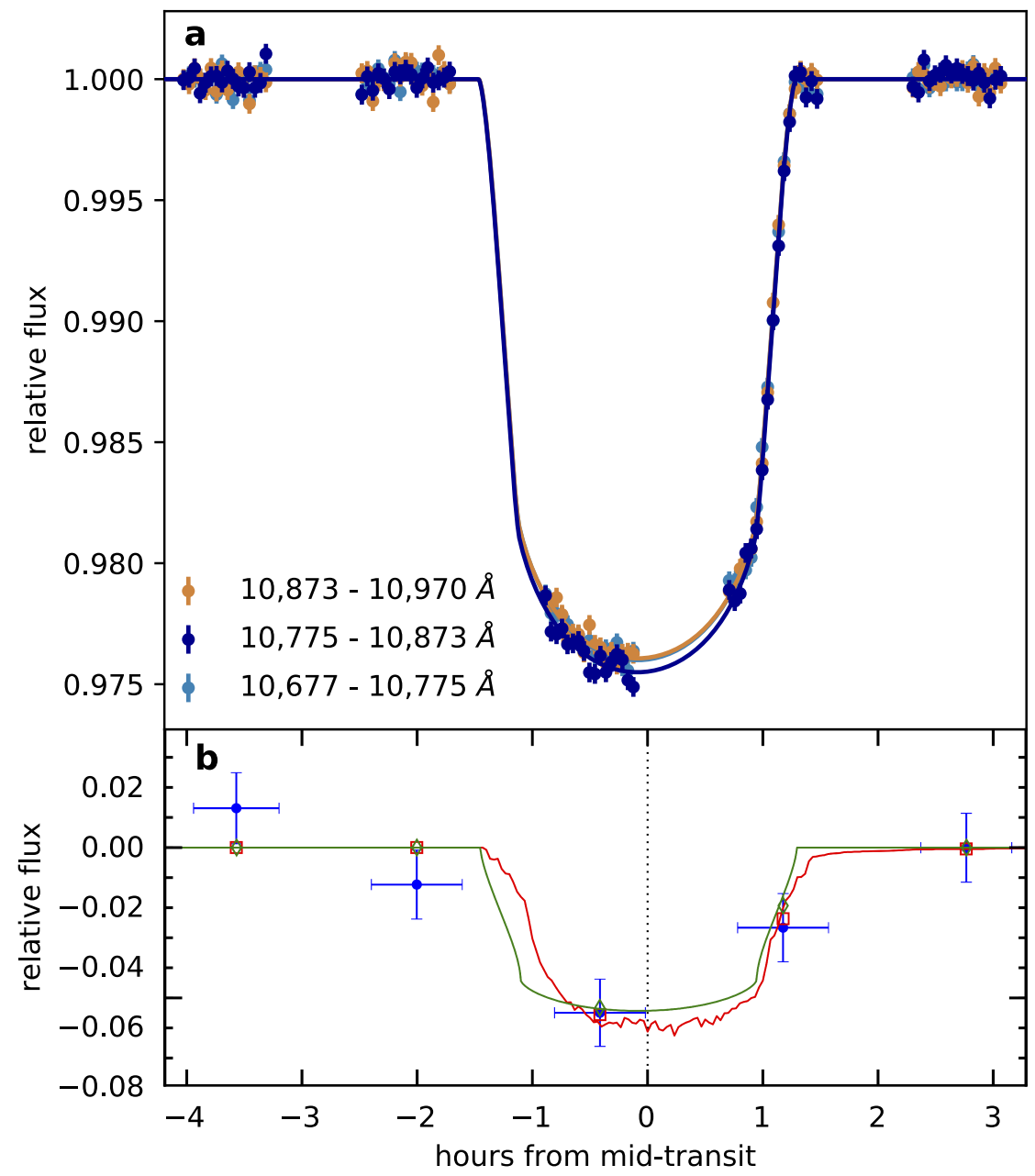

244 Figure 2 | Transit light curves for three $98 \AA$-wide spectroscopic channels. (a) Dark blue

245 points are from the channel centred on the He I 10,833 $\AA$ line, gold and light blue points are

246 from the two adjacent channels. All have $1 \sigma$ error bars. The transit depth of the blue light

247 curve is visibly deeper. (b) Binned difference between the 10,775 - 10,873 $\AA$ channel light

248 curve, and the average of the two adjacent channels (blue points, $1 \sigma$ errors), highlighting the

249 excess absorption. It is well explained by both our 1D (green line) and 3D (red line) escaping

250 atmosphere models. 


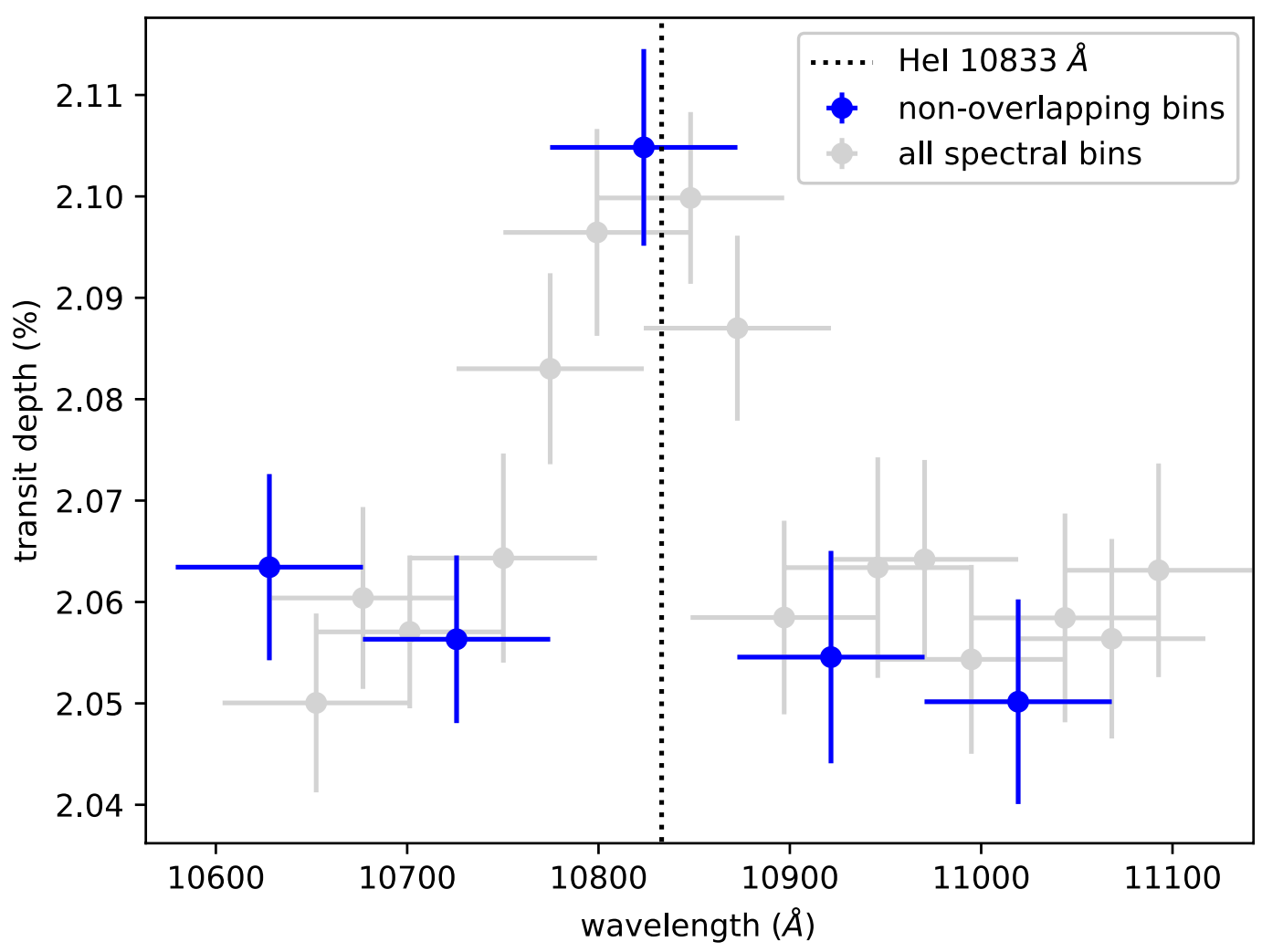

252

253 Figure 3 | Narrow-band transmission spectrum of WASP-107b, centred on 10,833 $\AA$.

254 Each spectroscopic channel has been shifted along one pixel from the last. Non-overlapping

255 bins are highlighted in blue. Error bars are $1 \sigma$. The peak of the spectrum coincides with the $256 \quad 2^{3} \mathrm{~S}$ helium absorption line at $10,833 \AA$. 

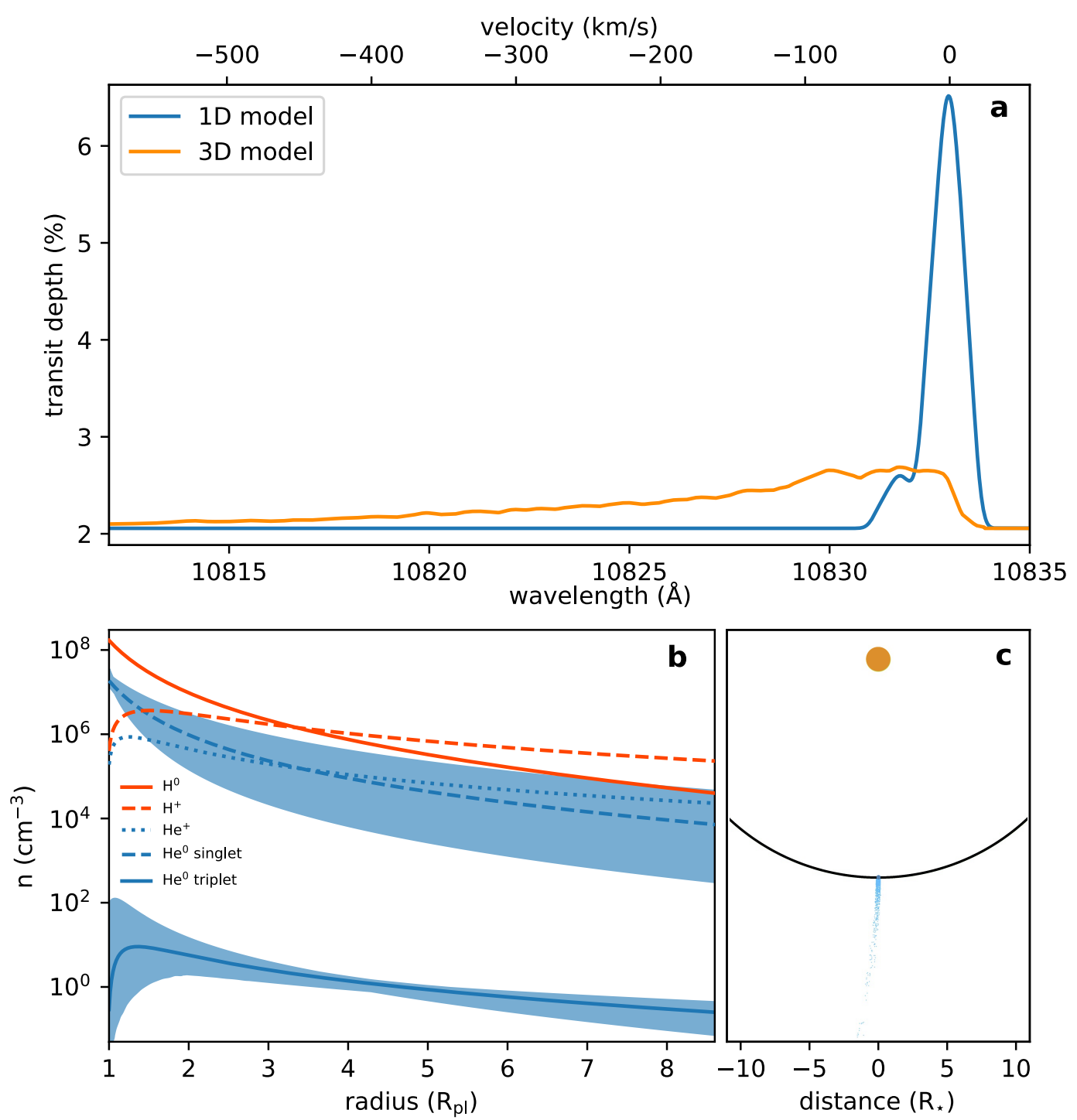

258 Figure 4 | Results from two models of WASP-107b's upper atmosphere. (a) Best-fit

259 absorption profiles of the helium $10,833 \AA$ triplet line from the 1-D (blue), and 3-D (orange)

260 models. Both reproduce the measured excess absorption of $0.049 \pm 0.011 \%$ in a $98 \AA$ bin.

261 Higher-resolution observations will resolve the profile shape, and further constrain the

262 velocity of the planetary wind. (b) Radial number density profiles of different atmospheric

263 species from the 1-D model, shaded blue regions are 1 $\sigma$ errors. (c) Top-down view of the

264 planetary system from the 3-D model, showing a comet-like tail of $2^{3} \mathrm{~S}$ helium shaped by

265 stellar radiation pressure. 


\section{Observations \& data reduction}

268 We observed one transit of WASP-107b with WFC3 in spectroscopic mode, using the G102 269 grism (GO-14916, P.I. Spake). This covers the approximate wavelength range of 8,780-

$27011,370 \AA$. We used forward spatial scanning to spread the spectra over $\sim 60$ pixels in the cross-dispersion direction with the SPARS10, NSAMP=15 setup, giving exposure times of $\sim 103$ seconds. This allowed 17 exposures per HST orbit. The observations lasted for five HST orbits, with two orbits pre-transit, one during the transit, and one post-transit, allowing us to precisely constrain the out-of-transit baseline.

The raw frames were first reduced with the automatic CalWF3 pipeline. The 1-D spectra were then extracted following standard methods ${ }^{30}$ : building up flux counts by summing the difference between successive non-destructive reads. We removed the background from each read difference by subtracting the median of a box of pixels uncontaminated by the spectrum. We found the flux-weighted centre of each scan and set to zero all pixels more than 75 rows away from the centre in the cross-dispersion axis, which removes many cosmic rays. The remaining cosmic rays were flagged by finding $4 \sigma$ outliers relative to the median along the dispersion direction. We replaced each flagged pixel with the median along the dispersion direction, re-scaled to the count rate of the cross-dispersion column. Since the scans are visibly tilted from the dispersion axis, we used the IRAF package Apall to fit the trace of the 2-D scans and extract 1-D spectra. We found the wavelength solutions by cross-correlating the extracted spectra with an ATLAS model stellar spectrum ${ }^{17}$ which most closely matches WASP-107 $\left(\mathrm{T}_{\text {eff }}=4,500 \mathrm{~K}, \log g=4.5 \mathrm{cgs}\right)$ modulated by the $\mathrm{G} 102$ grism throughput. 
291 We extracted the white light curve by summing the total counts of each 1-D spectrum. In order to constrain the mid-time of the transit, we fit the resulting time-series with the BATMAN transit model ${ }^{14}$, multiplied by a linear baseline trend and a physically-motivated systematics model. For the latter, we employed the RECTE model ${ }^{15}$, which accounts for two populations of charge traps in individual pixels of the detector and successfully replicates the ramp-like features that dominate the systematics. The RECTE model allows us to keep the first orbit of observations in our fit. The free parameters of our final model were: the planetto-star radius ratio, $R_{p} / R_{s}$; mid-transit time, $T_{0}$; the gradient and y-intercept of the linear background trend, $c_{1}$ and $c_{0}$ respectively; four parameters for the charge trapping model - the

300 initial number of populated slow and fast traps $s_{p o p}$ and $f_{p o p}$, and the changes in the two 301 populations between each orbit, $\delta$ s and $\delta f$; and an uncertainty rescaling factor, $\beta$ for the expected photon noise.

303 We fixed $a / R_{s}, i, e$, and the period using estimates from Kepler light curves ${ }^{16}$. To model the

304 stellar limb darkening we fitted a four-parameter non-linear limb darkening law ${ }^{31}$ to the

305 ATLAS stellar model described above.

306 Because the shape of the ramp-like systematics depends on the count level of the illuminated 307 pixels, the RECTE model requires the 'intrinsic' count rate of a pixel (i.e. the actual flux 308 received from the star) in order to model the charge trapping. To create a template of the 309 intrinsic count rate, we median-combined four raw images from the end of the second orbit.

310 Here the charge traps appear completely filled, and the ramp shape has tapered to a flat line.

311 It is possible to model each illuminated pixel, however, for a large scan this is

312 computationally expensive. Additionally, the ramp profile is washed out by systematics that 313 are introduced by telescope jittering and pointing drift. Instead we divided the scan into

314 columns of width 10 pixels along the dispersion axis and fed the median count profiles into 315 the model. 
316 We used the Markov chain Monte Carlo (MCMC) package emcee 32 to marginalise over the

317 parameter space of the model likelihood distribution. We used 80 walkers and ran chains for

3188000 steps, discarding the first 800 as burn-in before combining the walker chains into a

319 single chain. The best-fit model and residuals are shown in Extended Figure 1, with the

320 parameter values and $1 \sigma$ uncertainties reported in Extended Data Table 1. Although WASP-

$321107 \mathrm{~b}$ orbits an active star we see no evidence of star spot crossings. For context, only five

322 spot-crossing events are reported in 10 Kepler transits ${ }^{16,33}$.

323 Broadband spectroscopic light curve fit

324 We binned each spectrum into nine spectroscopic channels across the 8,780-11,370 $\AA$

325 wavelength range, each spanning 10-12 pixels on the detector. The resulting lightcurves are

326 shown in Extended Data Figure 2. Since the throughput of the G102 grism is wavelength-

327 dependent, the shape of the charge-trapping ramp in each spectroscopic light curve is

328 different. Therefore, for each channel we simultaneously fit for a transit model multiplied by

329 a linear baseline trend and a charge-trap model. To make a template of the intrinsic counts,

330 we took the median cross-dispersion-direction profile of each channel in the same four raw

331 images as used in the white light curve fit. We fixed $T_{0}$ to the value found from the white

332 light curve fit. Similarly to the white light curve fit, we fixed the orbital parameters to those

333 derived from Kepler light curves ${ }^{16}$, and wavelength-dependent limb darkening coefficients

334 from the ATLAS model. Therefore, for each channel the fitted parameters were

$335 R_{P} / R_{s}, c_{1}, c_{0}, s_{p o p}, f_{\text {pop }}, \delta s, \delta f$, and $\beta$. We ran MCMC fits for each light curve with emcee, with

33680 walkers, 80,000 steps and a burn-in of 800 .

337 As a test, we also ran additional fits for the spectroscopic light curves with the stellar limb

338 darkening coefficients as free parameters. This produced results that were consistent to within

$3391 \sigma$ with those obtained from the analysis in which the limb darkening coefficients were held

340 fixed. 
341 We show the resulting spectroscopic light curves divided by their best-fit systematics models

342 in Extended Data Figure 2, along with their residuals. Extended Data Table 2 reports our

343 median values for the transit depth, $\left(R_{P} / R_{S}\right)^{2}$, with $1 \sigma$ uncertainties calculated from the

344 MCMC chains. We also list the root mean square (RMS) of the residuals for each channel,

345 which range between 1.038-1.198 times the photon noise.

\section{Narrowband spectroscopic light curve fit around 10,830 angstroms}

347 To target the $10,833 \AA$ helium triplet, we binned the spectra from 10,590 to $11,150 \AA$ into

348 twenty narrowband channels. Each channel spanned 4 pixels on the detector, which is a

349 compromise between the low instrument resolution, signal-to-noise, and the narrowness of

350 the targeted feature. The wavelength coverage of each channel was shifted relative to the

351 adjacent channel by one pixel, so the channels overlap.

352 We note that since the formal resolution of the G102 grism is $\lambda / \Delta \lambda \sim 155$ at $10,400 \AA^{19}$

353 (which corresponds to $\Delta \lambda \sim 67 \AA$, or 2.7 pixel widths), the smallest bins theoretically

354 possible are 3 pixels wide. A resolution of 3 pixels could be achieved if the 10,833 $\AA$ feature

355 lay in the centre of a pixel, but in our data it lies significantly blue-ward of the centre of its

356 pixel. This means there is some $10,833 \AA$ flux in the pixel located two pixels blueward of the

$35710,833 \AA$ line. Indeed, when we tested the 3-pixel case we found that the amplitude of the

$35810,833 \AA$ feature increased by $0.011 \%$ from the 4-pixel-bin fit, which is similar to the

359 expected increase of $0.016 \%$ if all the $10,833 \AA$ flux fell within a central 3-pixel bin. With 3-

360 pixel bins the feature also appeared to have a slight blue 'wing', which is unlikely to be

361 astrophysical, as such wings would be expected from binning the data to a resolution higher

362 than that of the spectrograph. We therefore used conservative 4-pixel bins.

363 Extended Data Figure 3 shows the spectroscopic light curves divided by their best-fit

364 systematics models, along with their residuals. Extended Data Table 2 shows our median

365 values for the transit depth and their $1 \sigma$ uncertainties, calculated from the MCMC chains. We 
366 also list the RMS of the residuals of each channel, which range from 0.976 to 1.22 relative to

367 photon noise. The resulting transmission spectrum is shown in Figure 2.

368 Previous studies ${ }^{34}$ have highlighted the importance of considering the effect of stellar limb

369 darkening in stellar absorption lines on exoplanet transmission spectra. To investigate

370 whether this could cause the strong feature at $10,833 \AA$, we re-ran the narrow-band

371 spectroscopic light curve fits whilst fitting for a quadratic limb-darkening law. The resulting

372 spectrum was consistent with our previous analysis within 1-б.

373 Strong stellar lines that shift over the edges of pixels can introduce noise to measured

374 transmission spectra ${ }^{35}$. We checked this effect by smoothing our extracted time series spectra

375 with a Gaussian kernel of FWHM of 4 pixels, and re-running the narrowband spectroscopic

376 light curve fits. Our measured 10,833 $\AA$ absorption feature remained consistent within $1 \sigma$.

377 MEarth observations

378 Photometric monitoring observations were gathered using a single telescope of the MEarth-

379 South $^{36,37}$ array (CS 2015) at Cerro Tololo Inter-American Observatory (CTIO), Chile. Data

380 were obtained on 78 nights from 2017 March 22 (UT) to 2017 August 1 in groups of $4 \times 15$ s

381 exposures, with these exposure groups repeated at a cadence of approximately 30 minutes. A

382 total of 3096 exposures were gathered over this period. The bandpass of these observations is

383 in the red optical with the blue cutoff defined by RG715 glass at approximately 7,150 $\AA$ and

384 the red cutoff defined by the decline of the CCD quantum efficiency at approximately 10,000

$385 \AA$. For our data reduction, we used our previously published methodology ${ }^{38}$, modified for the

386 specifics of the MEarth data ${ }^{39}$.

387 The CCD camera shutter failed on 2017 May 9, which required removal for servicing.

388 This procedure introduces flat-fielding errors not corrected to sufficient precision by standard

389 calibrations, so instead we allow for this explicitly in the analysis by solving for a change in

390 the magnitude zero-points on both sides of the meridian at this date, following standard 
methods ${ }^{40}$. The result of this analysis is a "least-squares periodogram" (shown in Extended

Data Figure 4), obtained by simultaneously fitting a periodic modulation, while accounting

393 for the four magnitude zero-points and two additional linear terms describing sources of

394 systematic errors in the photometry (FWHM of the stellar images and the "common mode" as

395 a proxy for the effect of variable precipitable water vapor on the photometry). This procedure

396 would be mathematically equivalent to a Lomb-Scargle periodogram in the absence of these

397 six extra terms. The highest peak in the periodogram and its full width at half-maximum

398 corresponds to a periodicity of $19.7 \pm 0.9$ days. This is consistent with estimates from Kelper

399 light curves of $17.5 \pm 1.4$ days $^{33}$. We find an amplitude of $\sim 0.00150$ in magnitude.

400 AIT Photometry

401 We acquired nightly photometric observations of WASP-107 with the Tennessee State

402 University Celestron 14-inch (C14) automated imaging telescope (AIT) located at Fairborn

403 Observatory in southern Arizona ${ }^{41,42}$. The observations were made in the Cousins R passband

404 with an SBIG STL-1001E CCD camera. Differential magnitudes of WASP-107 were

405 computed with respect to eight of the most constant comparison stars in the CCD field.

406 Details of our data acquisition, reduction, and analysis can be found in a previous work ${ }^{43}$,

407 which describes a similar analysis of the planetary-host star WASP-31.

408 A total of 120 nightly observations (excluding a few observations in transit) were collected

409 between 2017 Feb. 23 and June 28. The nightly differential magnitudes are plotted in panel

410 (a) of Extended Data Figure 5. Panels (b) and (c) show the frequency spectrum of the

411 observations and the phase curve computed with the best frequency. Our frequency analysis

412 is based on least-squares sine fits with trial frequencies between 0.01 and $0.5 \mathrm{c} / \mathrm{d}$,

413 corresponding to periods between 2 and 100 days. The goodness of fit at each frequency is

414 measured as the reduction factor in the variance of the original data. Low-amplitude

415 brightness variability is seen at a period of $8.675 \pm 0.043$ days with a peak-to-peak amplitude 
416 of only 0.005 mag. Our period is almost exactly half the 17.5 -day rotation period found in

417 Kepler light curves ${ }^{33}$ and demonstrates that WASP-107 has spots or spot groups on opposite

418 hemispheres of the star during the epoch of our observations. The WASP-107b discovery

419 team $^{6}$ also found periods of around 17 and 8.3 days in their 2009 and 2010 photometry.

\section{Stellar variability correction}

421 To correct for stellar variability between the G141 and G102 epochs, we follow a similar

422 method to previous studies ${ }^{44,45}$, and estimate the flux from the non-spotted stellar surface as

$423 F_{s}=\max (F)+k \sigma$, where $F$ is the photometric light curve, $k$ is a fitted value and $\sigma$ is the

424 scatter of the light curve. A previous study ${ }^{44}$ found that $k=1$ is a good value to use for active 425 stars, so we adopt this value. We use the best-fit period, amplitude and ephemeris from the 426 MEarth photometry to estimate the expected flux dimming correction at the mid-transit times 427 for both data sets. We used the wavelength-dependent spot correction factor developed in a 428 previous work ${ }^{46}$ to correct for unocculted spots, and we set the spot temperature to be $3200 \mathrm{~K}$. 429 After the correction, the two spectra align well and appear to share a flat baseline. The one 430 overlapping spectral channel between G102/G141 is consistent within $1 \sigma$.

\section{$431 \quad$ ATMO retrieval}

432 For the combined G102 and G141 broadband spectrum corrected for photoshperic variability, 433 we performed an atmospheric retrieval analysis using our one-dimensional radiative transfer code, $\mathrm{ATMO}^{20,21,47,48,49}$. We assumed an isothermal temperature-pressure profile, and used MCMC to fit for the following parameters: atmospheric temperature; planetary radius at a pressure of 1 mbar; grey cloud opacity; and the abundances of $\mathrm{H}_{2} \mathrm{O}, \mathrm{CO}_{2}, \mathrm{CO}, \mathrm{CH}_{4}, \mathrm{NH}_{3}$, $\mathrm{H}_{2} \mathrm{~S}, \mathrm{HCN}$ and $\mathrm{C}_{2} \mathrm{H}_{2}$. We assumed solar abundances under chemical equilibrium for other gas species. Note that for this analysis we excluded wavelengths coinciding with the narrowband channel centred on the $10,833 \AA$ helium triplet. Our best-fit model is shown in Figure 1, with a a $\chi^{2}$ of 31.4 for 18 degrees of freedom. 
442 We checked that the residuals for the pixel columns in each frame do not reveal any obvious

443 anomalies over the narrow $10,833 \AA$ helium triplet, which suggests that it is not caused by a

444 detector defects or uncorrected cosmic rays. In addition, the transit depths remained

445 consistent within $0.5 \sigma$ when we removed $1 / 3$ of the points in the light curves, in several

446 random sub-sets, and re-fit them with the same procedures as described above.

\section{Absorption from other species}

448 The strong absorption line of metastable $2^{3} \mathrm{~S}$ helium at $10,833 \AA$ aligns extremely well with 449 the peak of the feature. In the $20 \AA$ region surrounding this peak $(10,820$ to $10,840 \AA)$,

450 helium is the only species that contains absorption solely within this wavelength range but nowhere else within the G102 bandpass ( 8,060 to $11,170 \AA$ ). There is, for example, a strong silicon absorption line at $10,830 \AA^{50}$, and a water line at $10,835 \AA$ (vacuum wavelengths) ${ }^{50}$, but if either species were the cause of the absorption seen in our transmission spectrum, there would be other similarly strong silicon lines measured at 10,588, 10,606 and 10,872 $\AA$, and a water line at 10,929 $\AA$, where we see no excess absorption. The other atoms with strong absorption lines near 10,833 Angstrom are $\mathrm{Np}, \mathrm{Cs}, \mathrm{Fe}, \mathrm{Th}, \mathrm{S}, \mathrm{Cr}, \mathrm{V}, \mathrm{Yb}$, and $\mathrm{Cu}-$ all of which can be ruled out as they are either radioactive with short half-lives, or have other strong transitions within the the 8,060 to $11,170 \AA$ wavelength range that we do not observe. We have also found there to be no species in the ExoMol ${ }^{51}$ or HITRAN/HITEMP ${ }^{52,53}$

460 databases with sufficiently sharp features aligned at 10,833 $\AA$. Specifically, we searched the 461 following species: $\mathrm{CH}_{4}, \mathrm{CO}_{2}, \mathrm{HCN}, \mathrm{NH}, \mathrm{CH}, \mathrm{OH}, \mathrm{PO}, \mathrm{NO}, \mathrm{VO}, \mathrm{TiO}, \mathrm{CN}, \mathrm{C}_{2}, \mathrm{PH}_{3}, \mathrm{NH}_{3}$, $\mathrm{SiO}, \mathrm{CaO}, \mathrm{H}_{3}+\mathrm{CO}, \mathrm{H}_{2} \mathrm{CO}, \mathrm{C}_{2} \mathrm{H}_{2}, \mathrm{BeH}, \mathrm{LiH}, \mathrm{HCl}, \mathrm{AlO}, \mathrm{SO}_{2}, \mathrm{H}_{2} \mathrm{~S}, \mathrm{PN}, \mathrm{KCl}, \mathrm{NaCl}, \mathrm{CS}, \mathrm{CP}$, PS, $\mathrm{MgH}, \mathrm{NaH}, \mathrm{CrH}, \mathrm{CaH}, \mathrm{FeH}$, and $\mathrm{ScH}$. We therefore conclude that absorption by metastable helium at $10,833 \AA$ is the most plausible explanation for the signal detected in the narrowband transmission spectrum. 


\section{Assessing the Earth's exosphere}

467 Where the Earth's exosphere is illuminated by XUV radiation from the sun, there is metastable helium. At an altitude of $\sim 500 \mathrm{~km}$, HST passes right through the Earth's exosphere, and when not in the Earth's shadow, will pass through regions containing metastable helium. The change in abundance of the metastable state throughout orbit has been shown to impart time-varying background signal in the $10,833 \AA$ line on the timescale of one $\sim 95$ minute spaceraft orbit ${ }^{54}$. There is no telluric metastable helium in Earth's shadow, and as expected, there is no significant excess absorption at 10,833 $\AA$ while HST is in Earth shadow $^{54}$. It does, however, affect HST measurements at dawn and dusk - i.e. when the spacecraft passes through the solar-illuminated upper atmosphere. The magnitude of the effect is correlated with the solar activity cycle - i.e. more activity, more UV, more metastable helium. The effect of spatially-diffuse telluric helium emission on WFC3 slitless spectroscopy is to impart an increased sky background signal across the detector. At the time of the observations, we were approaching solar minimum, and the $10.7 \mathrm{~cm}$ radiation (which is a proxy for solar activity) was only 70 solar flux units, sfu (Solar Monitoring Program,

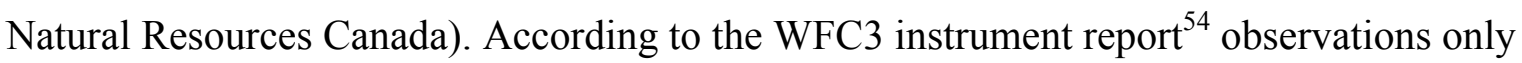
appear significantly affected when the $10.7 \mathrm{~cm}$ flux is greater than $\sim 100 \mathrm{sfu}$. Nonetheless, to test whether metastable helium at dawn and dusk in the Earth's atmosphere could cause an anomalous absorption feature in our transmission spectrum, we removed the first and last 4 exposures of each orbit - which encompasses the initial and final 10 minutes when HST passed through the illuminated dusk and dawn exosphere, and re-fit the light curves. The results were consistent with previous analysis at less than $1 \sigma$, which indicates that emission from telluric helium is not the cause of the narrowband absorption feature in our data. We note that previous transit spectroscopic studies using G102 $2^{55,56}$ do not show excess absorption at $10,833 \AA$. 


\section{Assessing the stellar chromosphere}

492 We also considered the possibility that the absorption feature we measure at $10,833 \AA$ could

493 be a result of stellar activity, since the metastable $2^{3} \mathrm{~S}$ state of helium is formed in the

494 inhomogeneous upper chromospheres and coronae of stars via photo-ionisation,

495 recombination, and collisional excitation. The planet passing over quiet regions with less

$49610,833 \AA$ helium absorption could in theory increase the relative transit depth at this

497 wavelength and thus mimic an exoplanet atmospheric feature.

498 Theoretical models of chromospheres ${ }^{57,58}$ predict the maximum equivalent width of the

49910,833 Angstrom helium line in the spectra of F- to early K-type stars to be $\sim 0.4 \AA$. Being a

500 K6 star, WASP-107 lies just outside the valid range of spectral types for this model.

501 However, in the following section we show that in order to match our observed transmission spectral feature, the nominal chromospheric absorption at $10,833 \AA$ of the WASP-107 host star would need to be five times stronger than any isolated (i.e. non-multiple), main-sequence

504 dwarf star measured to date.

505 After searching the literature for all 10,833 $\AA$ helium triplet equivalent width measurements

506 of isolated dwarf stars, we found over 300 measurements of over 100 distinct stars, including

50723 measurements of 11 different stars of similar spectral type to WASP-107 (K5-K7). We

508 found no measurements greater than $0.409 \AA^{59-64}$. We took an additional measurement of the

509 K6 star GJ380 with NIRSpec on Keck, which was found to have an equivalent width of 0.311

$510 \AA$ A (A. Dupree, private communication).

511 Furthermore, it has been shown ${ }^{55,63}$ that the equivalent width of the $10,833 \AA$ line is related to

512 that of another neutral helium absorption line, at 5,876 $\AA$. The 5,876 $\AA$ line is produced by

513 the transition from the $2^{3} \mathrm{D}$ to the $2^{3} \mathrm{P}$ state. As such, the $5,876 \AA$ line forms in the same

514 regions of the stellar chromosphere as the $10,833 \AA$ triplet (which corresponds to the $2^{3} \mathrm{~S}$ to 
$5152^{3} \mathrm{D}$ transition). Extended Data Figure 5 shows the equivalent width measurements of the

51610,833 and $5,876 \AA$ lines in a survey of 31 FGK stars ${ }^{63}$. A strong correlation is apparent.

517 To investigate the 5,876 ̊̊ helium line of WASP-107, we co-added high-resolution spectra

518 obtained with the HARPS spectrograph (ESO program 093.C-0474(A)). These spectra cover

519 a wavelength range of 3,800 to 6,900 $\AA$ (Extended Data Figure 5). We fit for the equivalent

520 width of the $5,876 \AA$ helium line in the co-added spectrum, with the result indicated on

521 Extended Data Figure 6 as a yellow shaded region. We find the equivalent width of this

522 feature is similar to that measured for other single dwarf stars, with no evidence of unusual

523 activity. Given the well-established correlation between the equivalent widths of the 5,876

524 and 10,833 $\AA$ helium lines noted above, this is further evidence against the WASP-107 host

525 star having an abnormally deep 10,833 Å line. In addition, we measured the S-index for

526 WASP-107 from the HARPS spectra, and found a night-averaged value of $\mathrm{S}_{\mathrm{HK}}=1.26 \pm 0.03$

527 (A.W., private communication), which is a moderate value for a $\mathrm{K} 6 \operatorname{star}^{64}$.

528 We therefore adopt the maximum equivalent width of $0.4 \AA$ to estimate an upper limit for the 529 amplitude of a feature that could be caused by un-occulted 10,833 $\AA$ helium absorption of

530 stellar origin in our 98 - $\AA$-wide spectroscopic channel. We consider the limiting case in

531 which WASP-107b occults only quiet regions of the star, where we assume there is no 10,833

$532 \AA$ absorption. This is the scenario in which the maximum amount of stellar continuum flux at

$53310,833 \AA$ would be blocked out by the planet, which we treat as a fully opaque disk. We

534 estimate the increased transit depth to be

$$
D_{\text {activity }}=\frac{A_{p l}}{1-\frac{W_{H e}}{W_{\text {bin }}}}=2.064 \pm 0.005 \%
$$

where $\mathrm{A}_{\mathrm{pl}}=2.056 \pm 0.005 \%$ is the fraction of the stellar area occulted by the planet; $\mathrm{W}_{\mathrm{He}}=0.4$

$537 \AA$, is the maximum equivalent width of the stellar absorption feature; and $\mathrm{W}_{\text {bin }}$ is the width of 538 the spectral bin (i.e. $98 \AA$ ). This gives an upper limit of the feature caused by stellar activity, 
$539 \delta \mathrm{D}_{\text {activity }}=\mathrm{D}_{\text {activity }}-\mathrm{A}_{\mathrm{pl}}=0.008 \pm 0.005 \%$, which is less than one fifth of the measured size of

540 the feature $(0.049 \pm 0.011 \%)$. We therefore conclude that the observed absorption feature

541 cannot be caused by stellar chromospheric spatial inhomogeneity alone.

\section{Resolution-Linked Bias}

543 If an absorption line overlaps in both a stellar and planetary atmosphere spectrum, and the

544 line is unresolved in the measured transmission spectrum, then the planetary absorption can

545 be underestimated. The effect is called Resolution Linked Bias (RLB) ${ }^{65}$. For the $10,833 \AA$

546 line in the WASP-107 system this dilution effect will compete with the possible over-

547 estimation of the signal from unocculted chromospherically active regions (as described in

548 the 'Assessing the stellar chromosphere' section). The magnitudes of both effects will depend

549 on whether the planet transits in front of active or quiet regions of the star. The RLB effect

550 would be largest if the planet transited only chromospherically active regions (which have the

551 highest 10,833 $\AA$ absorption). We estimated the magnitude of the RLB effect in this limiting

552 case following the method described in a previous work ${ }^{65}$, and assuming an equivalent width

553 of $0.4 \AA$ for the $10,833 \AA$ stellar line. For a measured absorption excess of $0.049 \pm 0.011 \%$ in a

$55498 \AA$ bin centred on the $10,833 \AA$ line, we could be underestimating the planetary absorption

555 by up to $0.009 \%$ (i.e. about one fifth of the measured signal). However, without knowledge

556 of which part of the chromosphere the planet transits; the stellar line profile; and the velocity

557 structure of the planetary helium signature, we cannot accurately estimate the magnitudes of 558 the competing effects.

\section{Stellar flares}

560 The He 10,833 $\AA$ line appears in emission in solar- (and presumably stellar-) flares ${ }^{66}$, so

561 active stars like WASP-107 could show short-term variability in the line, which may be

562 difficult to disentangle from a transiting planetary signal ${ }^{34}$. Flares are unlikely to wholly

563 mimic the signal we detect, since the planet would need to pass in front of flaring regions of 
564 the star throughout the duration of the transit. Instead, unocculted flares could dilute $\mathrm{He}$

$56510,833 \AA$ atmospheric absorption. Visual inspection of the raw light curve of the

566 spectroscopic channel centred on 10,833 $\AA$ shows no evidence of flare events. Additionally,

567 the pre- and post- transit flux levels agree with each other, which would not be the case if

568 there was significant $10,833 \AA$ emission from the tail of a flare. As a precaution, we re-

569 produced the narrowband transmission spectrum around the 10,833 $\AA$ line using different

570 combinations of the out-of transit baseline: firstly with only orbits 2 and 4, then with orbits 1

571 and 3, and then orbits 2 and 5. All three cases gave a 10,833 $\AA$ absorption feature consistent

572 to within $1 \sigma$ of our full fit.

573 Photospheric spots and faculae

574 To quantify the effect of a heterogeneous photosphere on the transmission spectrum around

$57510,833 \AA$, we used a variability modelling method ${ }^{67,68}$ which uses an ensemble of model

576 stellar photospheres with randomly located active regions to provide estimates of the fraction

577 of the stellar surface covered by photospheric spots and faculae for a given rotational

578 variability amplitude. While variability monitoring traces only the non-axisymmetric

579 component of the stellar heterogeneity and thus provides a lower limit on active region

580 covering fractions ${ }^{68}$, this numerical approach provides a more complete understanding of the

581 range of covering fractions that may correspond to an observed variability level. The model

582 describes the integrated full-disk spectrum by the combination of three components: the

583 immaculate photosphere, spots, and faculae. We used three spectra interpolated from the

584 PHOENIX model grid $^{69}$ with $\log g=4.5$ and $[\mathrm{M} / \mathrm{H}]=+0.02$ and different temperatures to

585 represent the three components. Following previous works ${ }^{68}$, we set the photosphere

586 temperature, $\mathrm{T}_{\text {phot, }}$, to the effective temperature of the star $\left(\mathrm{T}_{\text {eff }}=4430 \mathrm{~K}^{6}\right)$ and adopt scaling

587 relations for the spot temperature $\mathrm{T}_{\text {spot }}{ }^{70,71}$ and faculae temperature $\mathrm{T}_{\mathrm{fac}}{ }^{72}$. 
Thus, the temperatures of the three components are $T_{p h o t}=T_{\text {eff }}=4,430 \mathrm{~K}, T_{\text {spot }}=0.73 \times T_{\text {phot }}$

$589=3,230 \mathrm{~K}$, and $T_{f a c}=T_{p h o t}+100 \mathrm{~K}=4,530 \mathrm{~K}$. WASP-107b's discovery paper ${ }^{6}$ reports a 17-

590 day periodic modulation with a $0.4 \%$ semi-amplitude ( $0.8 \%$ full-amplitude) for WASP-107.

591 Assuming a typical spot radius of $r_{s p o t}=2^{\circ}$, we find the reported rotational variability could

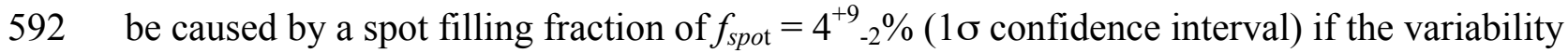

593 is due to spots alone. In the more realistic case in which spots and faculae are both

594 contributing to the variability, we find $f_{\text {spot }}=8_{-3}^{+6} \%$ and $f_{\text {faculae }}=53_{-12}^{+15} \%$. The covering

595 fractions we report are means over the entire model photosphere. They do not take into

596 account relative over- or under-abundances of magnetic features on the Earth-facing

597 hemisphere during a transit. Therefore, in the worst case scenario, they could underestimate

598 the hemispheric covering fractions by a factor of 2 . However, the $1-\sigma$ confidence intervals,

599 which are derived from 100 model realizations with randomly selected active region

600 locations, are deliberately conservative to account for this. Extended Data Figure 6 shows

601 how unocculted photospheric stellar heterogeneities could affect the transmission spectrum,

602 assuming the planet transits a chord of immaculate photosphere. The stellar contamination

603 factor, $\varepsilon$, on the y-axis is multiplied by the true $\left(R_{p} / R_{s}\right)^{2}$ transit depth to produce the observed

604 transmission spectrum, i.e. $\varepsilon>1$ means the observed transit depth is deeper than expected

605 from the planetary atmosphere model. The spots+faculae model does not predict an increase

606 in transit depth at 10,833 $\AA$. No sharp features around 10,833 $\AA$ are apparent. Instead, the

607 model predicts transit depths should be inflated by $\sim 1 \%$ across the full wavelength range of

608 G102 with perhaps some features apparent at $~ 8,500 \AA$ and 8,900 Angstrom (for this reason

609 we only use the 8,780-11,370 Å region in our full transmission spectrum, even though the

610 G102 throughput extends down to 8,000 $\AA$ ). The strong absorption feature we measure is

611 therefore unlikely to be caused by photospheric inhomogeneity.

\section{1-D escaping atmosphere model}


613 Here we give a brief overview of the first model used to investigate the narrowband

614 transmission spectrum at 10,833 $\AA$, which is presented and described in more detail in a

615 previous work ${ }^{22}$. This $1 \mathrm{D}$ model is based on the assumption that a thermosphere of a close-in

616 exoplanet can be well represented by the density and velocity profile of an isothermal Parker

617 wind driven by gas pressure ${ }^{73}$. We assume a composition of atomic hydrogen $(90 \%$ by

618 number) and helium (10\%). We find the solution for the hydrogen ionization balance and the

619 distribution of helium atoms in the ground, excited $2^{3} \mathrm{~S}$, and ionized states. The physical

620 processes taken into account in the helium balance are photoionization from the ground and

$6212^{3} \mathrm{~S}$ states, recombination to the singlet and triplet states, collisional transitions between the

622 triplet $2^{3} \mathrm{~S}$ state and states in the helium singlet ladder, which includes collisions with both

623 free electrons and neutral hydrogen atoms, and the radiative decay from the $2^{3} \mathrm{~S}$ state to the

624 ground state. The photoionization rates are calculated using the UV stellar flux of a K6 star

625 HD 85512 taken from the MUSCLES survey ${ }^{74}$ (version $2.1^{75,76}$ ), placed at the orbital distance

626 of WASP $107 \mathrm{~b}$ The equations used to compute the hydrogen/helium distributions, along with

627 all the relevant reaction rate coefficients and cross sections, are described in a previous

628 work $^{22}$. We only changed the input parameters such as the mass and radius of the planet and

629 its host star, as well as the input stellar spectrum, so that they match the properties of WASP

$630107 b$.

631 Based on the obtained density profile of helium in the $2^{3} \mathrm{~S}$ state, we calculate the optical

632 depth and the in-transit absorption signal at 10,833 $\AA$, assuming that a planet with a

633 spherically symmetric thermosphere transits across the center of the stellar disk. For a planet

634 of given mass and radius, the wind temperature and the total mass loss rate are free

635 parameters in the model. Based on the results from the literature ${ }^{77,78}$, we explore a

636 temperature range between 5,000-13,000 K. In order to produce the absorption signal

637 consistent with our measurement, the required mass loss rate is between $10^{10}$ and $3 \times 10^{11} \mathrm{~g} / \mathrm{s}$. 


\section{3-D escaping atmosphere model}

639 Our second model has previously been used to interpret the escaping exosphere of the

640 Neptune-mass exoplanet, GJ436 $\mathrm{b}^{9,23}$. It considers neutral helium atoms that are released from

641 the top of the thermosphere and subjected to planetary and stellar gravity, radiation pressure,

642 and photoionization. We found that the data are well explained by $2^{3} \mathrm{~S}$ helium atoms escaping

643 at a rate of $10^{6}-10^{7} \mathrm{~g} / \mathrm{s}$. Stellar radiation pressure on the escaping helium atoms is stronger

644 than the counter-balancing stellar gravity by a factor of approximately 10 and 50 for the

645 weakest and strongest of the $10,833 \AA$ triplet lines, respectively. Thus the gas blows away so

646 swiftly as to form a tail nearly aligned with the star-planet axis.

647

648 Code availability

649 The custom code used to extract the HST spectra from the raw data frames is available upon

650 request. The HST light curve fitting was performed using the open source BATMAN

651 (https://github.com/lkreidberg/batman) and emcee codes (http://github.com/ dfm/emcee), and

652 the proprietary RECTE code. The ATMO code used to compute the lower atmosphere

653 models is currently proprietary, as are the 1-D and 3-D upper atmosphere codes.

\section{Data availability}

655 Raw HST data frames are publicly available online at the Mikulski Archive for Space

656 Telescopes (MAST; https://archive.stsci.edu).

657

\section{References}

659 30. Evans, T. M., et al., Detection of $\mathrm{H} 2 \mathrm{O}$ and Evidence for TiO/VO in an Ultra-hot

660 Exoplanet Atmosphere, Astrophys. J., 822, L4 (2016) 
661 31. Claret, A., A new non-linear limb-darkening law for LTE stellar atmosphere models.

662 Calculations for $-5.0<=\log [\mathrm{M} / \mathrm{H}]<=+1,2000 \mathrm{~K}<=$ Teff $<=50000 \mathrm{~K}$ at several surface

663 gravities, Astron. Atrophys., 363, 1081-1190 (2000)

664 32. Foreman-Mackey, D., Hogg, D. W., Lang, D., Goodman, J., emcee: The MCMC

665 Hammer, PASP, 925, $306(2013)$

666 33. Močnik, T., Hellier, C., Anderson, D. R., Clark, B. J. M., Southworth, J., Starspots on

667 WASP-107 and pulsations of WASP-118, Mon. Not. R. Astron. Soc., 469, 1622-1629 (2017)

668 34. Czesla, S., Klocová, T., Khalafinejad, S., Wolter, U., Schmitt, J. H. M. M., The center-to-

669 limb variation across the Fraunhofer lines of HD 189733. Sampling the stellar spectrum using

670 a transiting planet, Astron. Atrophys., 582, 51 (2015)

671 35. Deming, D., et al., Infrared Transmission Spectroscopy of the Exoplanets HD 209458b

672 and XO-1b Using the Wide Field Camera-3 on the Hubble Space Telescope, Astrophys. J.,

673774, A95 (2013)

674 36. Nutzman, P., Charbonneau, D., Design Considerations for a Ground-Based Transit

675 Search for Habitable Planets Orbiting M Dwarfs, PASP, 120, 317 (2008)

676 37. Irwin J. M. et al., The MEarth-North and MEarth-South Transit Surveys: Searching for

677 Habitable Super-Earth Exoplanets Around Nearby M-dwarfs, Proc. Conf. 767, 18th

678 Cambridge Workshop on Cool Stars, Stellar Systems, and the Sun ed G. van Belle and H. C.

679 Harris (2014)

680 38. Irwin, J. et al., The Monitor project: rotation of low-mass stars in the open cluster M34,

681 Mon. Not. R. Astron. Soc., 370, 954 (2006)

682 39. Berta, Z. K. et al., Transit Detection in the MEarth Survey of Nearby M Dwarfs: Bridging

683 the Clean-first, Search-later Divide, Astron. J, 144, 145 (2012)

684 40. Irwin, J. et al., On the Angular Momentum Evolution of Fully Convective Stars: Rotation

685 Periods for Field M-dwarfs from the MEarth Transit Survey, eprint arXiv1011.4909 
686 41. Henry, G. W., Techniques for Automated High-Precision Photometry of Sun-like Stars,

687 PASP, 761, 845-860 (1999)

688 42. Eaton, J. A., Henry, G. W., \& Fekel, F. C. 2003, in The Future of Small Telescopes in the 689 New Millennium, Volume II - The Telescopes We Use, ed. T. D. Oswalt (Dordrecht:

$690 \quad$ Kluwer), 189

691 43. Sing, D. K., et al., HST hot-Jupiter transmission spectral survey: detection of potassium 692 in WASP-31b along with a cloud deck and Rayleigh scattering, Mon. Not. R. Astron. Soc., $693446,2428-2443(2015)$

694 44. Aigrain, S., Pont, F., Zucker, S., A simple method to estimate radial velocity variations 695 due to stellar activity using photometry, Mon. Not. R. Astron. Soc.., 419, 314 (2012)

696 45. Huitson, C. et al., An HST optical-to-near-IR transmission spectrum of the hot Jupiter 697 WASP-19b: detection of atmospheric water and likely absence of TiO, Mon. Not. R. Astron. 698 Soc., 434, 3252-3274 (2013)

699 46. Sing, D. K. et al., Hubble Space Telescope transmission spectroscopy of the exoplanet 700 HD 189733b: high-altitude atmospheric haze in the optical and near-ultraviolet with STIS, 701 Mon. Not. R. Astron. Soc., 416, 1443-1455 (2011)

702 47. Tremblin, P. et al., Cloudless Atmospheres for L/T Dwarfs and Extrasolar Giant Planets, 703 Astrphys. J., 817, L19 (2016)

704 48. Wakeford, H. R. et al., HAT-P-26b: A Neptune-mass exoplanet with a well-constrained 705 heavy element abundance, Science, 356, 628-631 (2017)

706 49. Evans, T. M. et al., An ultrahot gas-giant exoplanet with a stratosphere, Nature, 548, 58$707 \quad 61(2017)$ 
50. Kramida, A. et al., NIST Atomic Spectra Database (version 5.5.1), [Online]. Available: https://physics.nist.gov/asd [Tue Dec 05 2017]. National Institute of Standards and Technology, Gaithersburg, MD.

51. Tennyson, J. et al., The ExoMol database: molecular line lists for exoplanet and other hot atmospheres, J. Mol. Spectrosc., 327, 73-94 (2016)

52. Gordon, I. E. et al., The HITRAN 2016 molecular spectroscopic database, J. Quant. Spectrosc. Radiat. Transf., 203, 3-69 (2017)

53. Rothmans, L. S. et al., HITEMP, the high-temperature molecular spectroscopic database, J. Quant. Spectrosc. Radiat. Transf., 111, 2139-2150 (2010)

54. Brammer, G., Pirzkal, N., McCullough, P., MacKenty, J., Time-varying Excess Earthglow Backgrounds in the WFC3/IR Channel, Instrument Science Report WFC3 2014-03, Hubble Space Telescope, Space Telescope Science Institute, STScIc (2014) 55. Kreidberg et al., A Detection of Water in the Transmission Spectrum of the Hot Jupiter WASP-12b and Implications for Its Atmospheric Composition, Astrophys. J., 814, A15 (2015)

56. Wakeford, H. et al., The Complete transmission spectrum of WASP-39b with a precise water constraint, eprint arXiv:1711.10529

55. Andretta, V. \& Giampapa, M. S., A method for estimating the fractional area coverage of active regions on dwarf F and G stars, Astrophys. J, 439, 405 (1995)

57. Andretta, V. \& Jones, H. P., On the Role of the Solar Corona and Transition Region in the Excitation of the Spectrum of Neutral Helium, Astrophys. J., 489, 375 (1997) 58. Vaughan, A. H., Zirin, H., The Helium Line $\lambda 10830 \AA \AA$ in Late-Type Stars, Astrophys. J., $152,123(1968)$

59. Zirin, H., 10830 A He I observations of 455 stars, Astrophys. J., 260, 655 (1982) 
732

733

734

735

736

737

738

739

740

741

742

743

744

745

746

747

748

749

750

751

752

753

754

755

756

60. Zarro, D. M., Zirin, H., The dependence of He I 10830 A absorption strength upon X-ray emission in late-type stars, Astrophys. J., 304, 365 (1986)

61. Sanz-Forcada, J., Dupree, A. K., Active cool stars and He I $10830 \AA \AA$ : the coronal connection, Astron. Astrophys., 488, 715 (2008)

62. Takeda, Y., Takada-Hidai, M., Chromospheres in Metal-Poor Stars Evidenced from the He I 10830Å Line, PASJ, 63, 547 (2011)

63. Andretta, V., Giampapa, M. S., Covino, E., Reiners, A., Beeck, B., Estimates of Active Region Area Coverage through Simultaneous Measurements of the He I $\lambda \lambda 5876$ and 10830 Lines, Astrophys. J., 839, 97 (2017)

64. Isaacson, H. I., Fischer, D., Chromospheric activity and jitter measurements for 2630 stars on the California planet search, Astrophys. J., 725, 875-885 (2010).

65. Deming, D. \& Sheppard, K., Spectral Resolution-linked Bias in Transit Spectroscopy of Extrasolar Planets, Astrophys. J., 841, L3 (2017)

66. Li, H., You, J., Yu, X., Du, Q., Spectral Characteristics of Solar Flares in Different Chromospheric Lines and Their Implications, Solar Phys., 241, 301-315 (2007)

67. Rackham, B. et al., ACCESS I: an optical transmission spectrum of GJ 1214b reveals a heterogeneous stellar photosphere. Astrophys. J, 834, 151R (2017).

68. Rackham, B. V., Apai, D., Giampapa, M. S. The transit light source effect: false spectral features and incorrect densities for M-dwarf transiting planets, Astrophys. J, 853, 122 (2018). 69. Husser, T.-O. et al. A new extensive library of PHOENIX stellar atmospheres and synthetic spectra. Astron. \& Astrophys., 533A, 6H (2013).

70. Beryugina, S. Starspots: a key to the stellar dynamo. Living Reviews in Solar Physics, 2, $8(2005)$

71. Afram, N. \& Beryudina, S. Molecules as magnetic probes of starspots. Astronomy \& Astrophysics. 576A, 34A (2015). 
757 72. Gondoin, P. Contribution of Sun-like faculae to the light-curve modulation of young

758 active dwarfs. Astronomy \& Astrophysics. 478, 883G (2008).

759 73. Parker, E. N., Dynamics of the interplanetary gas and magnetic fields, Astrophys. J., 128,

$760 \quad 664(1958)$

761 74. France, K., et al., The MUSCLES Treasury Survey. I. Motivation and Overview,

762 Astrophys. J, 820, A89 (2016)

763 75. Youngblood, A., et al., The MUSCLES Treasury Survey. II. Intrinsic LY $\alpha$ and Extreme

764 Ultraviolet Spectra of K and M Dwarfs with Exoplanets*, Astrophys. J., 824, A101 (2016)

765 76. Loyd, R. O. P., The MUSCLES Treasury Survey. III. X-Ray to Infrared Spectra of 11 M

766 and K Stars Hosting Planets, Astrophys. J., 824, A102 (2016)

767 77. Lecavelier des Etangs, A., Vidal-Madjar, A., McConnell, J. C., Hebrard, G., Atmospheric 768 escape from hot Jupiters, Astron. \& Astrophys, 418, L1-L4 (2004)

769 78. Salz, M., Czesla, S., Schneider, P., C., Schmitt, J., H., M., M., Simulating the escaping

770 atmospheres of hot gas planets in the solar neighborhood, Astron. Astrophys, 586, A75

$771 \quad(2016)$ 


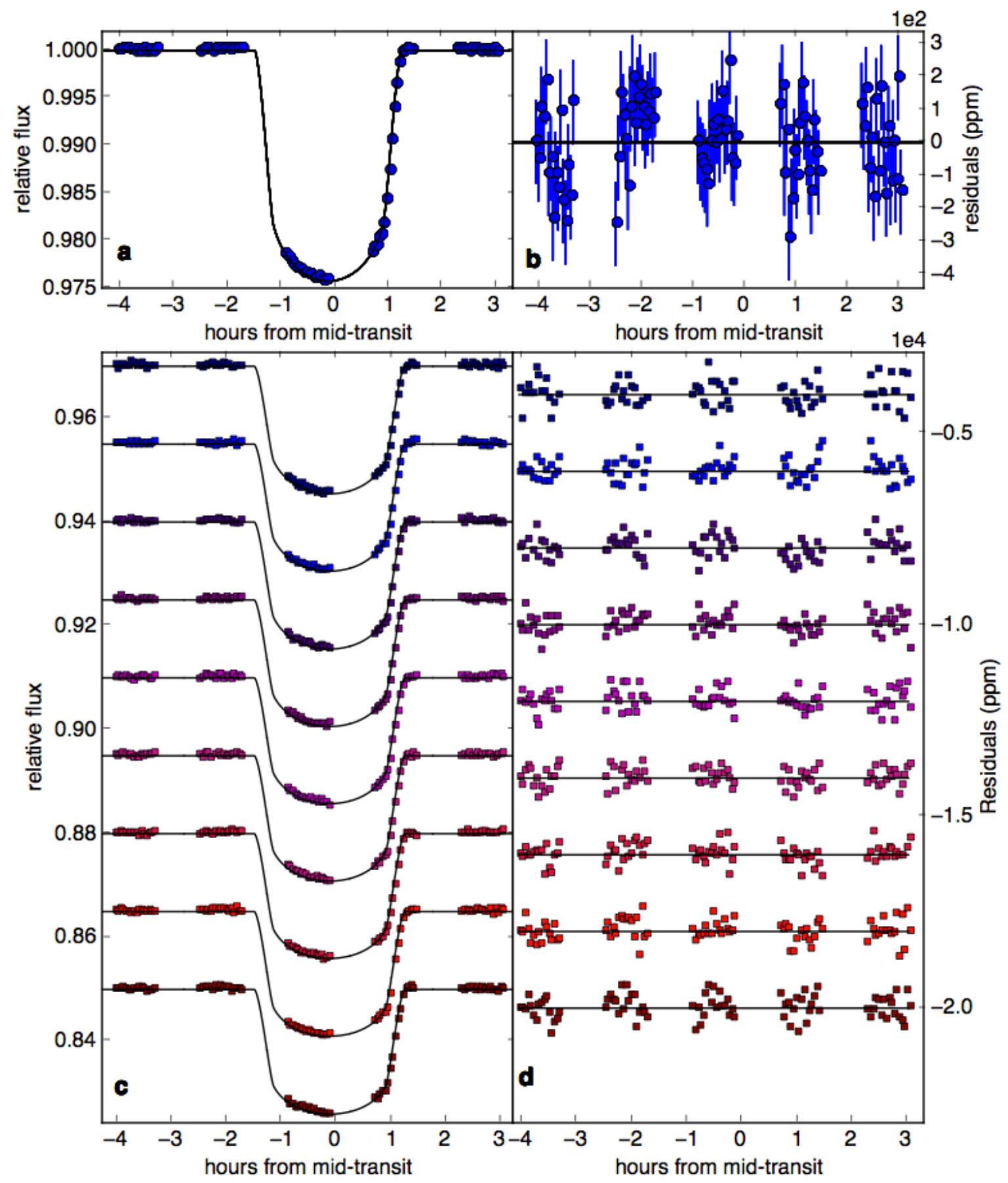

773 Extended Data Figure 1 | G102 white light curve and broadband spectroscopic light

774 curves covering the $\mathbf{0 . 8 8}-1.14$ micron wavelength range for WASP-107b. (a) White light

775 curve relative flux divided by systematics model, with best-fit transit light curve plotted in

776 black. (b) White light residuals and $1 \sigma$ errors, after removing the combined transit and 

clarity.
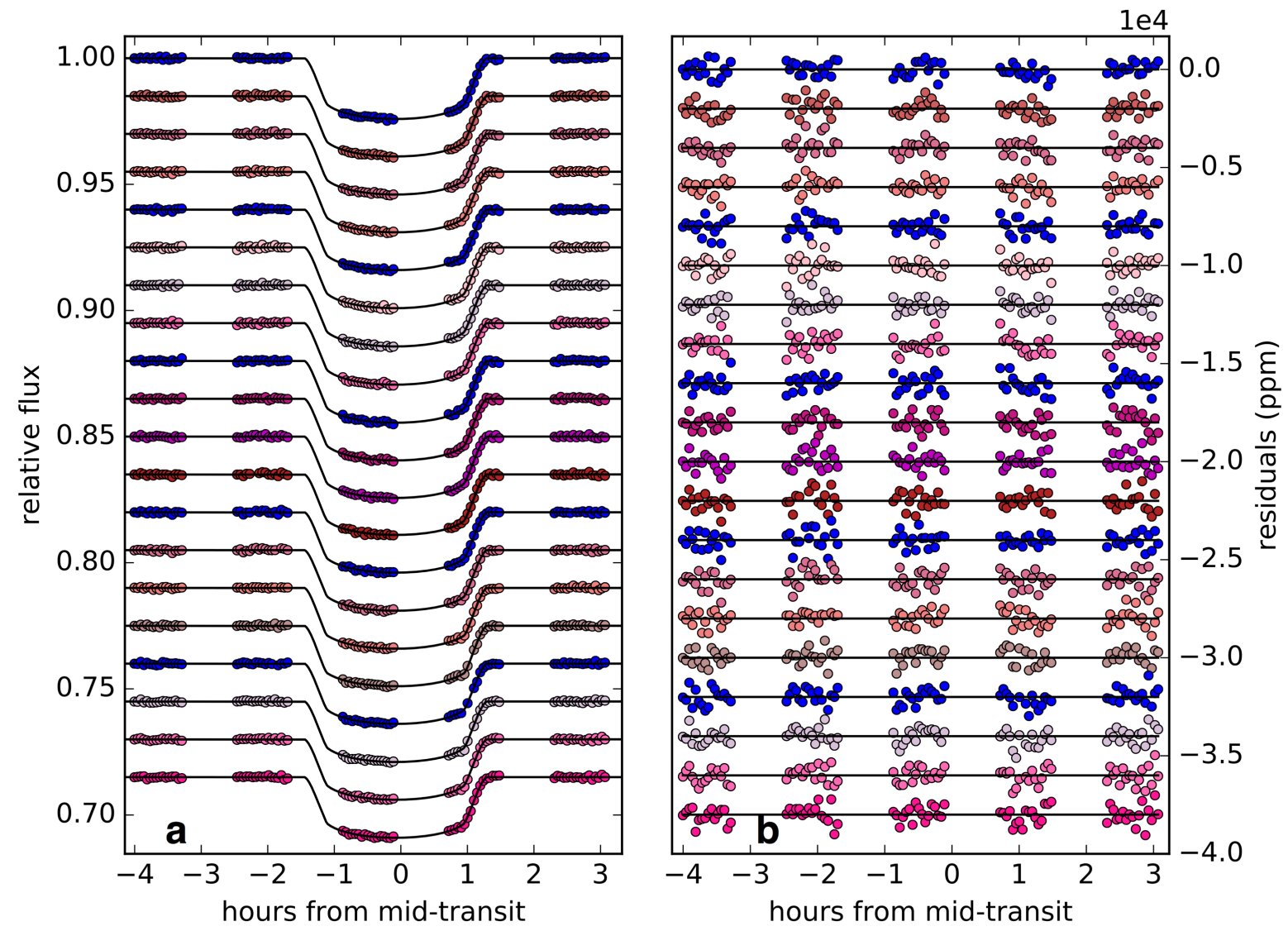

782 Extended Data Figure 2 | Narrow-band (4-pixel-wide) spectroscopic light curves

covering the 1.06-1.12 micron wavelength range. (a) Points are light curves divided by

systematics models, black curves are best-fit transit models. (b) Best-fit model residuals with absorption are highlighted in blue. 

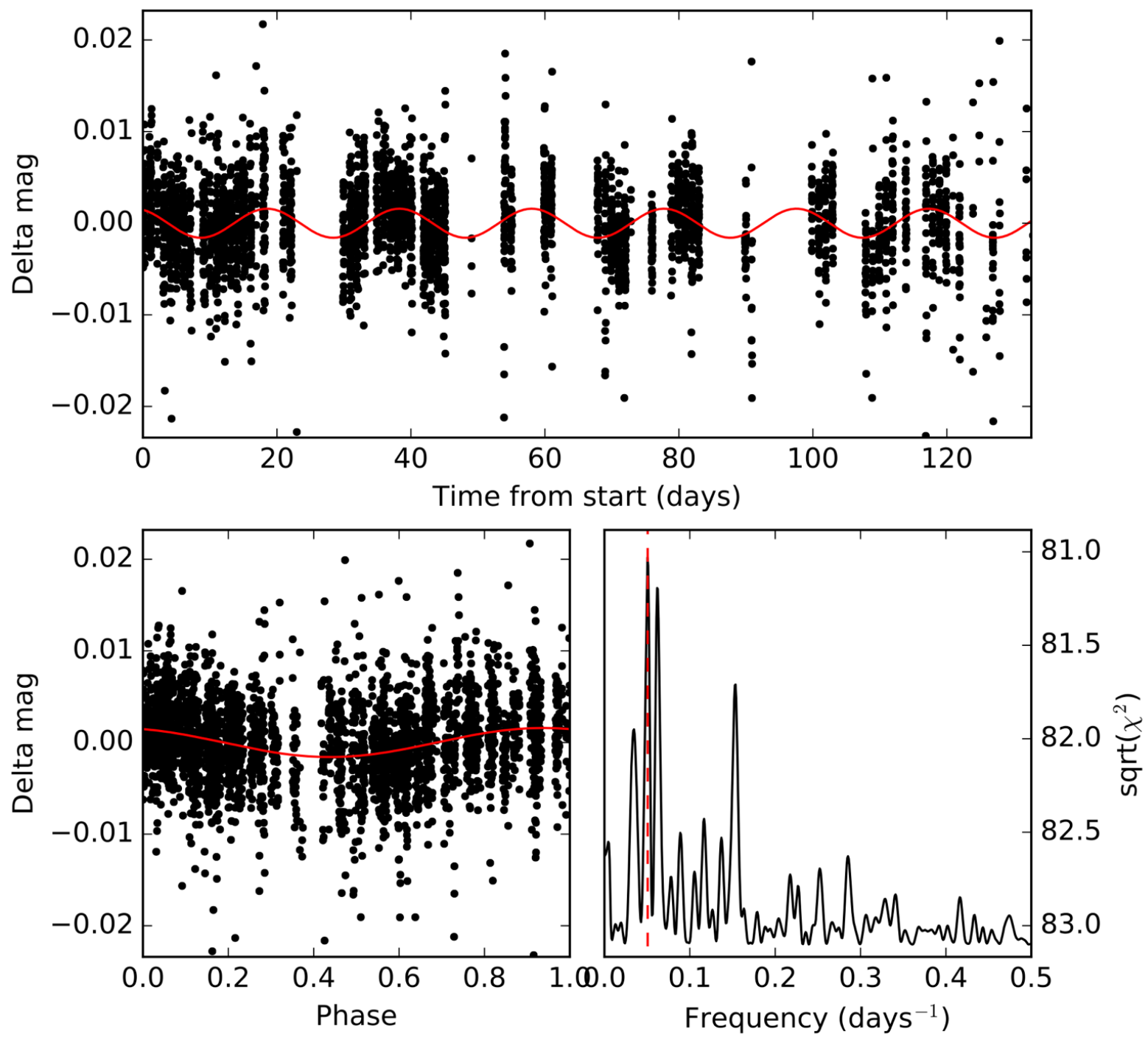

787

788 Extended Data Figure 3 | Ground-based photometry for WASP-107 from MEarth. We

789 performed a Lomb-Scargle periodogram search and found a best-fit period of $19.7 \pm 0.9$ days,

790 with a relative amplitude of $\sim 0.00150$ mag. 

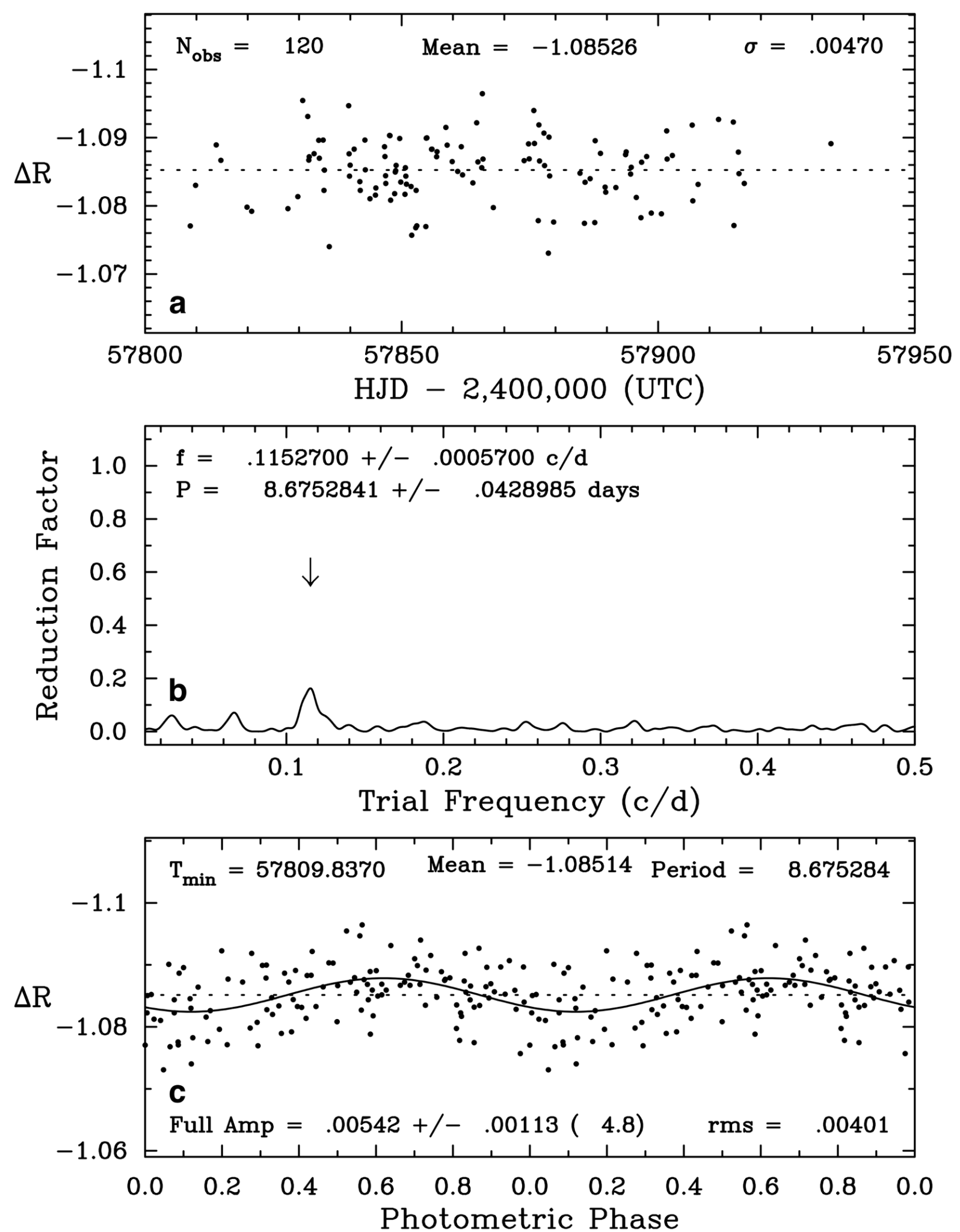
Photometric Phase

792 Extended Data Figure 4 | Ground-based photometry for WASP-107b from AIT. (a) The

793 nightly photometric observations of WASP-107 in the Cousins R band acquired with the

794 Tennessee State University C14 automated imaging telescope at Fairborn Observatory during 795 the 2017 observing season. (b) The frequency spectrum of the 2017 observations shows low- 

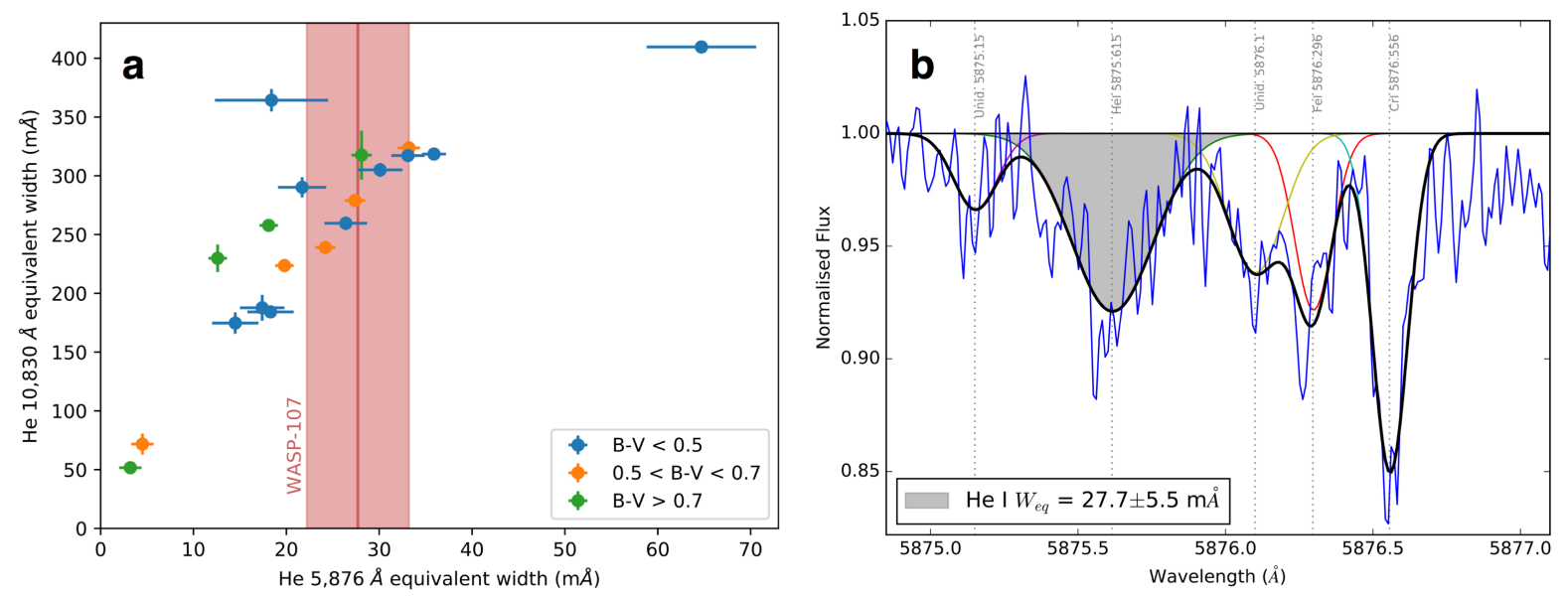

800 Extended Data Figure 5 | Equivalent widths of helium 5,876 $\AA$ and 10,830 $\AA$ lines. (a)

801 Measurements for 30 stars of different colour indices, from a previous work ${ }^{63}$. These two helium lines are expected to form in the same regions of stellar atmospheres and their equivalent widths are clearly correlated. Our 5,876 ^ measurement for WASP-107 is plotted

804 as a red line. Red shaded region shows the $1 \sigma$ error. Equivalent width measurement and $1 \sigma$ error of the 5,876 $\AA$ line for WASP-107 (B-V > 0.7) from HARPS spectra is shown as red shaded region. (b) Co-added spectra from HARPS radial velocity campaign for WASP-107

807 around the 5,876 ̊̊ line of metastable helium. Lines fit with Gaussian profiles. 

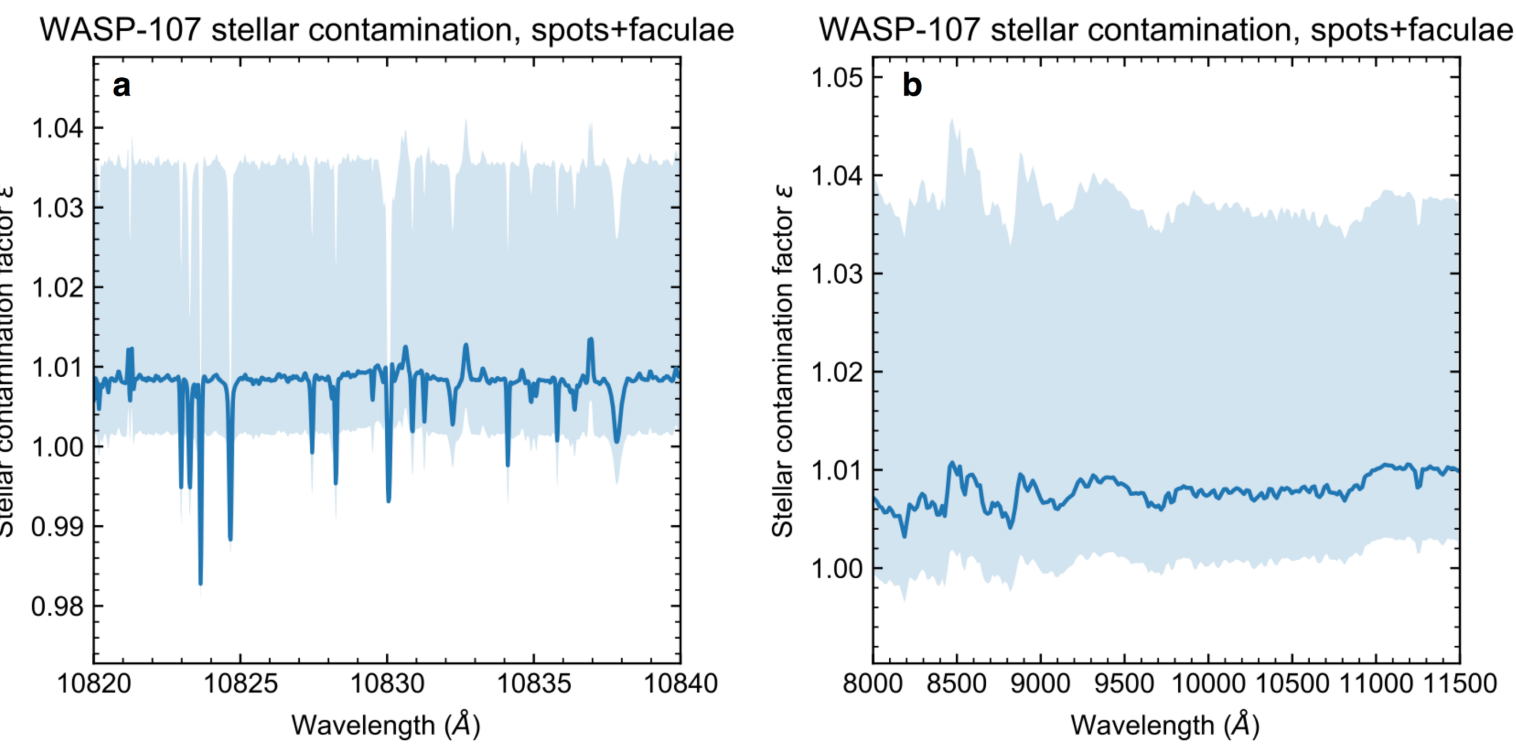

808

Extended Data Figure 6 | The effects of an inhomogeneous photosphere on the

810 transmission spectrum of WASP-107b. Lines show the stellar contamination produced by

811 unocculted spots and faculae. Shaded regions indicate the $1 \sigma$ uncertainty on the stellar

812 contamination due to the uncertainty on spot and faculae covering fractions. (a) The region

813 around the $10,830 \AA$ (air wavelength) helium triplet at the resolution of the PHOENIX

814 spectra $(\mathrm{R}=500,000)$. (b) The full G102 wavelength range in $15 \AA$ bins.

815

816

817

818 


\begin{tabular}{|c|c|}
\hline Parameter & Value \\
\hline$R_{P} / R_{S}$ & $0.142988 \pm 0.00012$ \\
\hline to (BJ Dutc) & $2,457,904.7295 \pm 0.0002$ \\
\hline$c_{0}$ & $1.00004 \pm 2 e-5$ \\
\hline$c_{1}$ & $-0.0018 \pm 0.0002$ \\
\hline Spop & $62 \pm 17$ \\
\hline$f_{P Q P}$ & $42 \pm 6$ \\
\hline$\delta s$ & $-2 \pm 10$ \\
\hline$\delta f$ & $65 \pm 4$ \\
\hline$\beta$ & $1.73 \pm 0.15$ \\
\hline$P$ & $5.72147^{a}$ \\
\hline$i\left(^{\circ}\right)$ & $89.7^{3}$ \\
\hline$a / R_{s}$ & $18.164^{a}$ \\
\hline e(assumed) & 0 \\
\hline
\end{tabular}

820 Extended Data Table 1 | Fitted parameters from the G102 white light curve. Errors

821 quoted encompass $68 \%$ of the MCMC samples after burn-in. (a) Parameters fixed from Dai 822 \& Winn (2017). 


\begin{tabular}{|c|c|c|c|c|c|}
\hline Wavelength (A) & $\begin{array}{l}\text { Transit } \\
\text { depth }(\%)\end{array}$ & Error (\%) & RMS (PPM) & RMS/ phot. & $\begin{array}{l}\text { Correction } \\
\text { factor }\end{array}$ \\
\hline $8,769-9,063$ & 2.0451 & 0.0084 & 326 & 1.178 & 1.007101 \\
\hline $9,063-9,356$ & 2.0425 & 0.0069 & 276 & 1.077 & 1.006785 \\
\hline $9,356-9,650$ & 2.0514 & 0.0079 & 285 & 1.184 & 1.006549 \\
\hline $9,650-9,943$ & 2.0514 & 0.0064 & 252 & 1.083 & 1.006454 \\
\hline $9,943-10,237$ & 2.0456 & 0.0066 & 264 & 1.167 & 1.006340 \\
\hline $10,237-10,530$ & 2.0448 & 0.0058 & 241 & 1.080 & 1.006303 \\
\hline $10,530-10,775$ & 2.0431 & 0.0065 & 245 & 1.048 & 1.006162 \\
\hline $10,873-11,142$ & 2.0461 & 0.007 & 269 & 1.152 & 1.006123 \\
\hline $11,142-11,386$ & 2.0509 & 0.0069 & 298 & 1.198 & 1.005945 \\
\hline $10,579-10,677$ & 2.0634 & 0.0091 & 344 & 0.989 & 1.00596 \\
\hline $10,604-10,701$ & 2.0500 & 0.0088 & 381 & 1.102 & 1.005923 \\
\hline $10,628-10,726$ & 2.0604 & 0.0089 & 366 & 1.061 & 1.006214 \\
\hline $10,652-10,750$ & 2.0571 & 0.0075 & 336 & 0.976 & 1.006167 \\
\hline $10,677-10,775$ & 2.0563 & 0.0082 & 360 & 1.043 & 1.006131 \\
\hline $10,701-10,799$ & 2.0643 & 0.0103 & 395 & 1.143 & 1.006046 \\
\hline $10,726-10,824$ & 2.0830 & 0.0094 & 354 & 1.023 & 1.005985 \\
\hline $10,750-10,848$ & 2.0964 & 0.0102 & 415 & 1.198 & 1.005928 \\
\hline $10,775-10,873$ & 2.1048 & 0.0097 & 391 & 1.126 & 1.005923 \\
\hline $10,799-10,897$ & 2.0998 & 0.0084 & 387 & 1.117 & 1.005948 \\
\hline $10,824-10,922$ & 2.0870 & 0.0091 & 390 & 1.128 & 1.005949 \\
\hline $10,848-10,946$ & 2.0585 & 0.0095 & 409 & 1.183 & 1.006008 \\
\hline $10,873-10,970$ & 2.0546 & 0.0104 & 385 & 1.111 & 1.005982 \\
\hline $10,897=10,995$ & 2.0634 & 0.0108 & 423 & 1.220 & 1.005973 \\
\hline $10,922-11,019$ & 2.0642 & 0.0098 & 377 & 1.087 & 1.005967 \\
\hline $10,946-11,044$ & 2.0543 & 0.0093 & 363 & 1.046 & 1.005935 \\
\hline $10,970-11,068$ & 2.0502 & 0.0101 & 375 & 1.084 & 1.005962 \\
\hline $10,995-11,093$ & 2.0584 & 0.0103 & 373 & 1.082 & 1.005918 \\
\hline 11,019 - 11,117 & 2.0564 & 0.0098 & 385 & 1.117 & 1.005897 \\
\hline $11,094-11,142$ & 2.0631 & 0.0105 & 414 & 1.197 & 1.005891 \\
\hline \multicolumn{6}{|c|}{$\begin{array}{l}\text { Modified Kreidberget } \\
\text { al. (2017) results: }\end{array}$} \\
\hline $11,210-11,450$ & 2.0723 & 0.0059 & & & 1.003979 \\
\hline $11,450-11,710$ & 2.0814 & 0.0055 & & & 1.003919 \\
\hline $11,710-11,960$ & 2.0585 & 0.0056 & & & 1.003918 \\
\hline $11,960-12,220$ & 2.0577 & 0.0054 & & & 1.003848 \\
\hline $12,220-12,480$ & 2.0535 & 0.0059 & & & 1.003892 \\
\hline $12,480-12,720$ & 2.0572 & 0.0050 & & & 1.003897 \\
\hline $12,720-12,980$ & 2.0699 & 0.0052 & & & 1.003830 \\
\hline $12,990-13,230$ & 2.0818 & 0.0050 & & & 1.003805 \\
\hline $13,230-13,490$ & 2.0742 & 0.0057 & & & 1.003983 \\
\hline $13,490-13,740$ & 2.0943 & 0.0048 & & & 1.004081 \\
\hline $13,740-14,010$ & 2.0878 & 0.0048 & & & 1.004059 \\
\hline $14,010-14,250$ & 2.0974 & 0.0052 & & & 1.004110 \\
\hline $14,250-14,520$ & 2.0907 & 0.0052 & & & 1.004126 \\
\hline $14,520-14,760$ & 2.0777 & 0.0051 & & & 1.004136 \\
\hline $14,760-15,020$ & 2.0767 & 0.0069 & & & 1.004107 \\
\hline $15,020-15,280$ & 2.0762 & 0.0057 & & & 1.004020 \\
\hline $15,280-15,520$ & 2.0593 & 0.0060 & & & 1.004116 \\
\hline $15,520-15,790$ & 2.0562 & 0.0064 & & & 1.004007 \\
\hline $15,790-16,030$ & 2.0581 & 0.0056 & & & 1.003941 \\
\hline $16,030-16,290$ & 2.0595 & 0.0065 & & & 1.003969 \\
\hline
\end{tabular}


824 Extended Data Table 2 | All results from transit light curve fits. Modified results from a

825 previous study ${ }^{18}$ are included. RMS is the root mean squared of the model residuals in parts

826 per million (PPM); the second-to-last column is the RMS divided by the expected photon

827 noise; the last column is the correction factor we applied to account for stellar variability.

\begin{tabular}{|l|l|}
\hline Parameter & $\begin{array}{l}\text { Limits from } \\
\text { MCMC }\end{array}$ \\
\hline Temperature $(\mathrm{K})$ & $650^{+120}{ }_{-80}$ \\
\hline Rp/Rs at Imbar & $0.914^{+0.010}-0.014$ \\
\hline VMR $\log _{10}\left(\mathrm{H}_{2} \mathrm{O}\right)$ & $-1.7^{+03}{ }_{-0.6}$ \\
\hline VMR $\log _{10}\left(\mathrm{CO}_{2}\right)$ & $<10$ \\
\hline VMR $\log _{10}(\mathrm{CO})$ & $<11$ \\
\hline VMR $\log _{10}\left(\mathrm{CH}_{4}\right)$ & $<10$ \\
\hline VMR $\log _{10}\left(\mathrm{NH}_{3}\right)$ & $<10$ \\
\hline VMR $\log _{10}\left(\mathrm{H}_{2} \mathrm{~S}\right)$ & $<11$ \\
\hline VMR $\log _{10}(\mathrm{HCN})$ & $<11$ \\
\hline VMR $\log _{10}\left(\mathrm{C}_{2} \mathrm{H}_{2}\right)$ & $<10$ \\
\hline
\end{tabular}

829 Extended Data Table 3 | Results from ATMO retrieval code for the lower atmosphere.

830 VMR stands for volume mixing ratio. Uncertainties for temperature, $\mathrm{R}_{\mathrm{p}} / \mathrm{R}_{\mathrm{s}}$ and VMR $\mathrm{H}_{2} \mathrm{O}$

831 encompass $68 \%$ of the MCMC samples after burn-in. Upper limits are from $1 \sigma \mathrm{MCMC}$

832 errors. 